ACCEPTED MANUSCRIPT

\title{
Guiding-centre theory for kinetic-MHD modes in strongly flowing plasmas
}

To cite this article before publication: Samuel Lanthaler et al 2019 Plasma Phys. Control. Fusion in press https://doi.org/10.1088/1361$\underline{6587 / a b 1 d 21}$

\author{
Manuscript version: Accepted Manuscript \\ Accepted Manuscript is "the version of the article accepted for publication including all changes made as a result of the peer review process, \\ and which may also include the addition to the article by IOP Publishing of a header, an article ID, a cover sheet and/or an 'Accepted \\ Manuscript' watermark, but excluding any other editing, typesetting or other changes made by IOP Publishing and/or its licensors" \\ This Accepted Manuscript is @ Ecole Polytechnique Federale de Lausanne.
}

During the embargo period (the 12 month period from the publication of the Version of Record of this article), the Accepted Manuscript is fully protected by copyright and cannot be reused or reposted elsewhere.

As the Version of Record of this article is going to be / has been published on a subscription basis, this Accepted Manuscript is available for reuse under a CC BY-NC-ND 3.0 licence after the 12 month embargo period.

After the embargo period, everyone is permitted to use copy and redistribute this article for non-commercial purposes only, provided that they adhere to all the terms of the licence https://creativecommons.org/licences/by-nc-nd/3.0

Although reasonable endeavours have been taken to obtain all necessary permissions from third parties to include their copyrighted content within this article, their full citation and copyright line may not be present in this Accepted Manuscript version. Before using any content from this article, please refer to the Version of Record on IOPscience once published for full citation and copyright details, as permissions will likely be required. All third party content is fully copyright protected, unless specifically stated otherwise in the figure caption in the Version of Record.

View the article online for updates and enhancements. 


\title{
Guiding-centre theory for kinetic-MHD modes in strongly flowing plasmas
}

\author{
S. Lanthaler ${ }^{1}$, J.P. Graves ${ }^{1}$, D. Pfefferlé2 ${ }^{2}$, and W.A. Cooper ${ }^{1}$ \\ ${ }^{1}$ Ecole Polytechnique Fédérale de Lausanne (EPFL), Swiss Plasma Center (SPC), CH-1015 \\ Lausanne, Switzerland \\ ${ }^{2}$ The University of Western Australia, 35 Stirling Highway, Crawley WA 6009, Australia
}

\section{April 15, 2019}

\begin{abstract}
A kinetic MHD model with kinetic pressure closure is derived from a consistent guiding-centre framework. Higher-order (gyroviscous) corrections to the pressure tensor are derived in complex geometry from a reduced kinetic equation. The proposed model allows for flows of the order of the thermal ion velocity, taking into account important centrifugal effects due to the $E \times B$ flow, as well as the effects of diamagnetic flows associated with finite Larmor radius corrections to both ion fluid inertia and long mean free path contributions. Wave-particle interactions, such as toroidal drift-resonance, are retained. Furthermore, the linearized model includes a quasi-neutrality equation, allowing the effects of a parallel electric field to be studied in fast rotating tokamak plasmas.
\end{abstract}

\section{Introduction}

Plasmas of relevancê to fusion research operate at high temperatures and low collisionality. The conditions found in such plasmas clearly place them outside the domain of validity of fluid models such as ideal magnetohydrodynamics (MHD), which are derived based on an assumption of high collisionality. Despite this fact, MHD has proven to be an indispensable and robust tool for the analysis of experiments and remains one of the main sources of understanding of macroscopic instabilities. This 'unreasonable effectiveness' of MHD eyen at high temperatures and corresponding long mean-free path lengths of ions, is commonly understood to arise as a consequence of the presence of a strong magnetic field; Perpendicular to the field lines, a fluid description may be justified, because the short mean-free path that is found in collisional regimes (allowing fluid closure in MHD) is effectively replaced by the small Larmor radius of particles even at low collisionality. However, parallel to the field lines the particles are free-streaming in strongly magnetized near-collisionless plasmas and kinetic effects such as resonant wave-particle interaction can become important. 
The study of collisionless, strongly magnetized plasmas was pioneered by Chew, Goldberger and Low [1]. In [1], an attempt was made to derive fluid equations based on an expansion in the parameter $\epsilon_{B} \sim \rho_{i} / L \ll 1$, where the Larmor radius of ions $\rho_{i}$ is assumed to be small relative to the characteristic length scales of the (macroscopic) plasma motion. As a result of the asymptotic expansion of moments of the Vlasov equation in $\epsilon_{B}$, it was found that the leading-order form of the pressure tensor is no longer isotropic, but instead is given in the so-called Chew-Goldberger-Low (CGL) form $\boldsymbol{P}=p_{\|} \boldsymbol{b} \boldsymbol{b}+p_{\perp}(\boldsymbol{I}-\boldsymbol{b} \boldsymbol{b})$. Here $\boldsymbol{b}$ is the unit vector pointing along the magnetic field, and $p_{\|}, p_{\perp}$ denote the parallel and perpendicular pressure components, respectively. The derivation in [1] does not lead to a closed set of fluid equations because the equations which determine $p_{\|}, p_{\perp}$ depend on heat fluxes which are not provided by the model. If these heat fluxes are simply neglected, the CGL double adiabatic fluid model is obtained, according to which the evolution of $p_{\|}$and $p_{\perp}$ is determined by [1]:

$$
\frac{d}{d t}\left(\frac{p_{\| B^{2}}}{\rho^{3}}\right)=0, \quad \frac{d}{d t}\left(\frac{p_{\perp}}{\rho B}\right)=0 .
$$

Here, $d / d t \equiv \partial / \partial t+\boldsymbol{u} \cdot \nabla$ is the total time derivative with $\boldsymbol{u}$ the fluid velocity, $B$ the magnetic field strength and $\rho$ denotes the mass density. The derivation of Chew, Goldberger and Low was restricted to lowest order in the $\epsilon_{B}$-expansion. It was subsequently recognized that finite Larmor-radius (FLR) effects can exert a stabilizing influence on plasmas. Such FLR effects are reflected by higher-order $\epsilon_{B}$-corrections to the pressure tensor and independent of collisionality. Among the early papers on this subject, Roberts and Taylor [2] have pointed out the importance of the additional (higher-order in $\epsilon_{B}$ ) components of the pressure tensor which persist in the limit of vanishing collisionality and are closely related to diamagnetic flows. These components form a tensor $\boldsymbol{\pi}_{\wedge}$ that is commonly referred to as the gyroviscous tensor. It was shown that the inclusion of the resulting gyroviscous force $\boldsymbol{F}_{\wedge} \equiv-\nabla \cdot \boldsymbol{\pi}_{\wedge}$ in the momentum equation leads to a gyroviscous cancellation, where $\boldsymbol{F}_{\wedge}$ approximately cancels out the effect of diamagnetic flows in the fluid inertia. A number of authors have subsequently extended the initial results of 2] to include additional effects, such as temperature variations and stress tensor drift [3, temperature gradients and higher-order moments of the distribution function [4], or the combined effects of collisions and FLR corrections [5, 6. Most of these results are obtained in simple slab geometry - a discussion of the assumptions required for the validity of this approximation has for example been given by Hazeltine and Meiss [7, Section 4.3.3]. In complex geometry, an expression for the gyroviscous pressure tensor has first been obtained under the fast flow ordering $\partial / \partial t \sim \boldsymbol{u} \cdot \nabla \sim v_{\mathrm{th}} / L$ by MacMahon [8], whose result remains the state-of-the-art in collisionless fluid theory under these assumptions. The findings of [8] have recently been confirmed by Ramos [9], who presents a comprehensive discussion of fluid models for collisionless plasmas including alternative orderings. In a later publication [10], an explicit expression for the gyroviscous force $\boldsymbol{F}_{\wedge}$ in complex geometry is also provided.

While the fluid moment approach provides useful information on extended fluid effects (diamagnetic flows, heat fluxes, higher-order moments), it does not provide a closed set of equations describing the dynamics collisionless plasmas, except under very particular circumstances which are not usually found [1]. In general, some form of ad hoc closure remains necessary. 
The present work focuses instead on an approach where a closure can be achieved from the solution of a reduced kinetic (guiding-centre or gyrokinetic) equation, taking into account both FLR as well as other kinetic corrections. Our approach shares some similarity with the one recently adapted by several authors in gyrokinetic theory [11, 12]. In the gyrokinetic approach, a dynamical reduction is first employed to transform the Vlasov equation to suitable gyro-centre coordinates, thereby eliminating the fast timescales associated with gyro-motion. The gyrokinetic reduction allows FLR effects to be retained under the ordering $k_{\perp} \rho_{i} \sim 1$, where the Larmor radius of ions is allowed to be of the same order of magnitude as the characteristic length scale of the perturbations. This comes at the expense of limiting consideration to small amplitude fluctuations of the electromagnetic fields about a long-wavelength background equilibrium.

The gyrokinetic derivation of reduced fluid equations including FLR corrections has first been proposed by Brizard [11. In [11, a set of gyrofluid equations has been obtained in gyrocentre coordinates and then transformed to physical particle space. Using this method, gyroviscous cancellations are automatically accounted for and extensions to arbitrary order in $k_{\perp} \rho_{i}$ can be systematically carried out. Building on these ideas, an expression for the non-linear gyroviscous force has subsequently been obtained by Belova [12]. The results of [12] are in agreement with the fluid results [4], but are only established for electrostatic perturbations and in slab geometry. To the knowledge of the authors, no derivation of FLR corrections to the pressure tensor is available in the existing literature from a reduced kinetic description and in complex geometry.

The present work fills this gap by giving a detailed derivation of the collisionless gyroviscous tensor in complex geometry. In contrast to [11, 12, we will follow the guiding-centre approach which allows for arbitrary amplitude perturbations, while requiring $k_{\perp} \rho_{i} \ll 1$. Strong flows are allowed for and full electromagnetic perturbations are retained. In this way, a guiding-centre kinetic-MHD model for strong flows including diamagnetic (FLR) effects is obtained. The pressure coupling approach is followed; closure of the momentum equation is achieved by expressing the pressure moment in terms of the solution of the guiding-centre equations. It is shown that the higher-order corrections to the Larmor motion that describe the deviation of a particle trajectory from circular motion around the magnetic field play a crucial role in determining the off-diagonal components of the pressure tensor. Our derivation naturally leads to a consistent hybrid kinetic-MHD description of collisionless plasmas in which the exact fluid equations are closed by approximate pressure moments obtained from the solution of a reduced kinetic equation. The proposed model accounts for kinetic effects such as Landau damping, includes an exact treatment of finite orbit-width effects and allows the investigation of strong flows and diamagnetic effects based on the consistent framework of guiding-centre theory. Special consideration is given to the kinetic-MHD equilibrium, and a set of equations suitable for the study of linear dynamics within the proposed model is derived. In particular, the model equations derived here generalise the results of Porcelli [13] and Antonsen, Lee [14 to include centrifugal effects, and FLR corrections in the fluid contributions to the plasma inertia.

The kinetic-MHD approach taken in the present work should be contrasted with gyrofluid [15. and two-fluid approaches [16], that have previously been used to investigate diamagnetic effects. Gyrofluid models are based on taking moments of the gyrokinetic equation, and therefore intrinsically rely on a splitting between background fields and small-scale fluctuations. Instead, we follow a more classical kinetic-MHD approach 
which does not require such a splitting. Our approach is instead restricted to the study of macroscopic, long-wavelength perturbations. Furthermore, our objective is not the derivation of a self-consistent set of fluid equations including FLR corrections for the study of small-scale turbulence. Rather, our goal is to discuss how several important, missing kinetic effects can be added to fluid descriptions of macroscopic instabilities. In particular, the present work concerns the interplay between the fluid and guidingcentre descriptions of plasmas, and shows how they can be combined in a consistent framework.

The present approach may more naturally be viewed as being complementary to a (two-)fluid approach, such as is for example implemented in the XTOR-2F code [17, 16]. Where instead of employing an ad hoc fluid closure, a kinetic closure is achieved, thus taking into account kinetic effects such as wave-particle resonances. As mentioned at the beginning of this introduction, a kinetic closure for the pressure is required at weak collisionality. Our work presents a model including both fluid effects and a reduced kinetic equation. It is explicitly shown that the kinetic description is consistent with two-fluid effects such as diamagnetic drifts. Our equations do however not form a superset of the two-fluid model employed by XTOR-2F. In particular, collisions and related diffusive processes are not included in our collisionless kinetic description.

This paper is organized as follows. In section 2 , a set of guiding-centre equations is developed in a local frame moving with a time-dependent background flow $\boldsymbol{u}(x, t)$. Important higher-order corrections due to gradients of the background flow are considered in detail in section 3.1. Before advancing to the general calculation of the pressure tensor including gyroviscous corrections, the discussion of section 3.1 focuses on a special case in simple geometry; this provides an intuitive picture of the meaning and origin of the gyroviscous stresses, and clearly relates them to deviations of particle trajectories from circular motion. Section 3.2 then presents our derivation of the general form of the gyroviscous pressure tensor from guiding-centre theory. The derivation is shown to recover MacMahon's results [8]. The full set of non-linear kinetic-MHD equations is discussed in Section 4.1. The kinetic-MHD equilibrium is considered in section 4.2 . Equations suitable for the computation of the linear kinetic response are derived in section 4.3. Combining the results of the current work, a linear kinetic-MHD model is finally obtained by extending the Frieman-Rotenberg equation [18] to include the kinetic equations of section 4.4. Further details on the calculations as well as several mathematical identities that may be of interest in other contexts are provided in the appendix.

\section{Guiding-centre equations in co-moving frame}

For the derivation of the FLR corrections to the pressure tensor, it will be useful to develop a set of guiding-centre equations based on the velocity variable decomposition $\boldsymbol{v}=\boldsymbol{u}(\boldsymbol{x}, t)+\boldsymbol{w}$, where $\boldsymbol{u}$ is the (leading-order in $\epsilon_{B}$ ) fluid velocity and $\boldsymbol{w}$ can be viewed as a thermal fluctuation. This has been achieved in [19] for the case of a timeindependent equilibrium flow $\boldsymbol{u}$. A similar approach has been followed in [20] in the case of time-varying fields, but a slightly different decomposition $\boldsymbol{v}=\boldsymbol{u}_{E}(\boldsymbol{x}, t)+\widetilde{\boldsymbol{w}}$ was employed, with $\boldsymbol{u}_{E}$ the $E \times B$ velocity. In this section, we extend the results in 19] to allow for time-varying fields. It turns out that the calculation is an almost

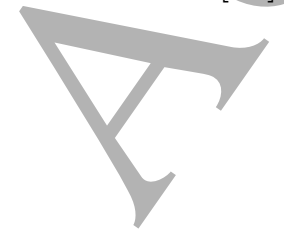


verbatim repetition of [20, appendix], with the only difference being that $\boldsymbol{u}_{E}$ is now replaced by $\boldsymbol{u}$ and the parallel velocity $\boldsymbol{v}_{\|}$is replaced by the fluctuating parallel part $w \boldsymbol{b}$ relative to $\boldsymbol{u}$. Along the way we have found two errors in [20, appendix], which do not affect the results in the main text of that work but which will be crucial for the present work ${ }^{1}$ This therefore justifies the presentation of relevant results provided in the current paper.

It is interesting to emphasize at this point that the gyroviscosity corrections to the pressure are analogous in their origin to the guiding-centre polarization corrections to the density moment and magnetization corrections to the velocity moment, respectively. Therefore, the subtle distinction between the higher-order displacement expressed in guiding-centre $\mathcal{Z}_{\text {gc }}$ versus physical particle coordinates $\mathcal{Z}_{\text {phy }}$, which has previously been pointed out and discussed in detail by Brizard [21] for the non-rotating $(\boldsymbol{u}=0)$ case, is important also in the present context. Even though the important distinction occurs in a second-order $\left(\epsilon_{B}^{2}\right)$ term when expressing the particle position $\boldsymbol{x}$ in terms of the guiding-centre position $\boldsymbol{X}$ (in guiding-centre variables) in the form

$$
\boldsymbol{x}=\boldsymbol{X}+\epsilon_{B} \boldsymbol{\rho}_{0}+\epsilon_{B}^{2} \boldsymbol{\rho}_{1}+O\left(\epsilon_{B}^{3}\right)
$$

the second-order correction $\boldsymbol{\rho}_{1}$ will play an important role in calculating the correct first-order $\epsilon_{B}$-correction to the pressure tensor. This perhaps perplexing fact can be understood as follows: The pressure moment is written in terms of the physical particle velocity $\boldsymbol{v}=\dot{\boldsymbol{x}}=\dot{\boldsymbol{X}}+\dot{\boldsymbol{\rho}}_{0}+\dot{\boldsymbol{\rho}}_{1}+\ldots$. While the higher-order guiding-centre displacement $\rho_{1}$ is ordered at $O\left(\epsilon_{B}^{2}\right)$, the velocity associated with the second-order displacement is $\dot{\boldsymbol{\rho}}_{1} \approx \Omega \partial_{\zeta} \boldsymbol{\rho}_{1}$. Because the gyrofrequency $\Omega \sim \epsilon_{B}^{-1}$ is a formally large term, it causes the velocity contribution due to $\boldsymbol{\rho}_{1}$ to be of order $\epsilon_{B}$ relative to $\dot{\boldsymbol{X}} \sim O(1)$; therefore, indicating the ordering in $\epsilon_{B}$ we find that

$$
\begin{aligned}
\dot{\boldsymbol{x}} & =\dot{\boldsymbol{X}}+\dot{\boldsymbol{\rho}}_{0}+\epsilon_{B} \dot{\boldsymbol{\rho}}_{1}+O\left(\epsilon_{B}^{2}\right) \\
& =\boldsymbol{W}+\epsilon_{B} \boldsymbol{V}_{\mathrm{gc}}+\dot{\boldsymbol{\rho}}_{0}+\epsilon_{B} \dot{\boldsymbol{\rho}}_{1}+O\left(\epsilon_{B}{ }^{2}\right),
\end{aligned}
$$

and $\epsilon_{B} \dot{\boldsymbol{\rho}}_{1}$ is of the same order of magnitude as the guiding-centre $\nabla B$ - and $\boldsymbol{\kappa}$-drifts contained in $\epsilon_{B} \boldsymbol{V}_{\mathrm{gc}}$ (defined in (8), below), and therefore needs to be retained when evaluating the particle velocity including $O\left(\epsilon_{B}\right)$-corrections.

Since our formulation deviates only slightly from the one adopted by Madsen [20], we will only cite the final results. We use the guiding-centre coordinates $(\boldsymbol{X}, w, \mu, \zeta)$, of which $\boldsymbol{X}, \mu$ and $\zeta$ are defined in the same way as in [20], but where we retain the leading-order parallel flow component of $\boldsymbol{u}$, so that $\boldsymbol{u}=\boldsymbol{u}_{\|}+\boldsymbol{u}_{E}$ and we will choose $\boldsymbol{u}_{\|}$ in a way that is suitable for the given application. Correspondingly, in our formulation the parallel velocity variable $w$ is chosen so that $\dot{\boldsymbol{X}}_{\|}=\boldsymbol{u}_{\|}(\boldsymbol{X}, t)+w \boldsymbol{b}(\boldsymbol{X}, t)$. We find that the guiding-centre Lagrangian is written

$$
\begin{aligned}
\mathcal{L} & =[q \boldsymbol{A}+m \boldsymbol{W}] \cdot \mathrm{d} \boldsymbol{X}+J \mathrm{~d} \zeta-H \mathrm{~d} t \\
H & =q \Phi+\frac{1}{2} m \boldsymbol{W}^{2}+\mu B
\end{aligned}
$$

${ }^{1}$ When passing from [20, eq. (A23)] to [20, eq.(A25)], the expression for the fluctuating part $\tilde{g}_{\mu}$ in [20, eq. (A24)] should be without the factor $\frac{1}{2}$ in front of the last term, and as a consequence the time derivative occurring in the expression for $s_{3}$ [20, eq. (A34)] should not have a factor $\frac{1}{2}$. Similarly, we should substitute $\frac{1}{2} \frac{\partial \boldsymbol{b}}{\partial t} \rightarrow \frac{\partial \boldsymbol{b}}{\partial t}$ in $\left[20\right.$, eq. (A35)]. Finally, $\hat{\boldsymbol{\theta}} \hat{\boldsymbol{\theta}}: \nabla(\ldots)$ should be replaced by $\frac{1}{2}(\hat{\boldsymbol{\theta}} \hat{\boldsymbol{\theta}}-\hat{\mathcal{\perp}} \hat{\perp}): \nabla(\ldots)$ in [20, eq. $(\mathrm{A} 34),(\mathrm{A} 35)]$ to ensure that the gyro-angle average of $s_{3}$ vanishes. 
with $\boldsymbol{W} \equiv w \boldsymbol{b}(\boldsymbol{X}, t)+\boldsymbol{u}(\boldsymbol{X}, t)$ the leading-order guiding-centre velocity and $J \equiv \frac{m}{q} \mu$ the gyroangle action. The phase-space transformation corresponding to this form of the guiding-centre Lagrangian is generated by the phase-space vector field $G=G_{1}+\epsilon_{B} G_{2}$ according to $\mathcal{Z}_{\mathrm{gc}}^{\alpha}=\mathcal{Z}_{\text {phy }}^{\alpha}+\epsilon_{B} G_{1}^{\alpha}+\epsilon_{B}^{2}\left(G_{2}^{\alpha}+\frac{1}{2} G_{1} \cdot \mathrm{d} G_{1}^{\alpha}\right)$. Following [21], the inverse transformation expressed in guiding-centre coordinates is given by

$$
\mathcal{Z}_{\text {phy }}^{\alpha}=\mathcal{Z}_{\mathrm{gc}}^{\alpha}-\epsilon_{B} G_{1}^{\alpha}-\epsilon_{B}^{2}\left(G_{2}^{\alpha}-\frac{1}{2} G_{1} \cdot \mathrm{d} G_{1}^{\alpha}\right)
$$

As pointed out above, for the purposes of the present work, the second-order correction to the spatial component of the guiding-centre transformation is required. Comparing (2) and (4), we can identify $\boldsymbol{x}=\boldsymbol{X}+\epsilon_{B} \boldsymbol{\rho}_{0}+\epsilon_{B}^{2} \boldsymbol{\rho}_{1}$, where

$$
\begin{aligned}
& \boldsymbol{\rho}_{0}=-G_{1}^{\boldsymbol{x}}, \\
& \boldsymbol{\rho}_{1}=-G_{2}^{\boldsymbol{x}}+\frac{1}{2} G_{1} \cdot \mathrm{d} G_{1}^{\boldsymbol{x}} .
\end{aligned}
$$

Following [21, 20, we fix a perpendicular frame $\boldsymbol{e}_{1}(\boldsymbol{X}, t), \boldsymbol{e}_{2}(\boldsymbol{X}, t)$ consisting of unit vectors such that $\boldsymbol{e}_{1}, \boldsymbol{e}_{2}, \boldsymbol{b}$ form a right-handed orthonormal basis at each point. The leading-order displacement is given in terms of the gyroangle-dependent unit vector $\widehat{\boldsymbol{\rho}}$ as

$$
\boldsymbol{\rho}_{0}(\boldsymbol{X}, \mu, \zeta, t)=\sqrt{\frac{2 \mu}{m B(\boldsymbol{X}, t)}} \hat{\boldsymbol{\rho}}(\boldsymbol{X}, \zeta, t),
$$

with $\widehat{\boldsymbol{\rho}}=\cos (\zeta) \boldsymbol{e}_{1}-\sin (\zeta) \boldsymbol{e}_{2}$. The leading order velocity is then along

$$
\widehat{\perp}(\boldsymbol{X}, \zeta, t) \equiv \partial_{\zeta} \widehat{\boldsymbol{\rho}}=-\sin (\zeta) \boldsymbol{e}_{1}(\boldsymbol{X}, t)-\cos (\zeta) \boldsymbol{e}_{2}(\boldsymbol{X}, t)
$$

After some algebraic manipulation detailed in the appendix, the higher-order displacement can be written

where

$$
\begin{gathered}
\boldsymbol{\rho}_{1}=-\left(g_{\mu} \partial_{\mu}+g_{\zeta} \partial_{\zeta}\right) \boldsymbol{\rho}_{0}-\frac{1}{\Omega}(\boldsymbol{b} \cdot \nabla \times \boldsymbol{W}) \boldsymbol{\rho}_{0} \\
-\left(G_{2, H}^{\boldsymbol{X}}+\frac{1}{2} \boldsymbol{\rho}_{0} \cdot \nabla \boldsymbol{b} \cdot \boldsymbol{\rho}_{0}\right) \boldsymbol{b} .
\end{gathered}
$$

$$
\begin{aligned}
g_{\mu} & =G_{1}^{\mu}-\mu \boldsymbol{\rho}_{0} \cdot \nabla \log B \\
g_{\zeta} & =G_{1}^{\zeta}+\boldsymbol{\rho}_{0} \cdot \boldsymbol{R},
\end{aligned}
$$

with $\boldsymbol{R} \equiv\left(\nabla \boldsymbol{e}_{1}\right) \cdot \boldsymbol{e}_{2}$. Explicit expressions for the components $g_{\mu}, g_{\zeta}$ are given by (55), (56) in appendix B.

Correcting an error in $[20]^{2}$, the parallel component $G_{2,||}^{\boldsymbol{X}}$ is found to be given by

$$
\begin{aligned}
G_{2, \|}^{\boldsymbol{X}}= & -\frac{1}{\Omega} \boldsymbol{\rho}_{0} \cdot \boldsymbol{b} \times\left[\boldsymbol{b} \cdot \nabla \boldsymbol{W}+\boldsymbol{W} \cdot \nabla \boldsymbol{b}+\partial_{t} \boldsymbol{b}\right] \\
& -\frac{\mu}{4 q \Omega}[\hat{\boldsymbol{\rho}} \widehat{\boldsymbol{\rho}}-\hat{\perp} \hat{\perp}]: \nabla \boldsymbol{b}
\end{aligned}
$$

${ }^{2}$ In contrast to [20, our calculation results in the second term gyroaveraging to 0 , while the term in (1) square bracket involves a convective derivative $\partial_{t} \boldsymbol{b}+\boldsymbol{W} \cdot \nabla \boldsymbol{b}$, as is required to ensure Galilean invariance. 
The guiding-centre equations of motion are obtained from variations of the Lagrangian (3) (cf. [20]). For the computation of the pressure, the following explicit expressions will be used

$$
\begin{aligned}
\dot{\boldsymbol{X}} & =\boldsymbol{W}+\epsilon_{B} \boldsymbol{V}_{\mathrm{gc}}+O\left(\epsilon_{B}^{2}\right) \\
\boldsymbol{V}_{\mathrm{gc}} & =\frac{\mu}{q B} \boldsymbol{b} \times \nabla B+\frac{\boldsymbol{b}}{\Omega} \times\left(\frac{\partial}{\partial t}+\boldsymbol{W} \cdot \nabla\right) \boldsymbol{W} \\
\dot{\zeta} & =\epsilon_{B}{ }^{-1} \Omega+\epsilon_{B}{ }^{0}\left[\boldsymbol{R} \cdot \boldsymbol{W}+S+\frac{1}{2} \boldsymbol{b} \cdot(\nabla \times \boldsymbol{b})\right]+O\left(\epsilon_{B}\right)
\end{aligned}
$$

Here $S$ is the "time-like" analogue of $\boldsymbol{R}$, namely $S=\left(\partial_{t} \boldsymbol{e}_{1}\right) \cdot \boldsymbol{e}_{2}$.

\section{Pressure in guiding-centre coordinates}

\subsection{Intuitive picture in slab geometry}

To provide an intuitive account of the guiding-centre corrections described in the last section, we focus here on the particle motion and implications of these corrections in a simplified magnetic geometry. Following [5], we assume a cartesian slab geometry described by coordinates $\left(x_{1}, x_{2}, x_{3}\right)$ and constant magnetic field $\boldsymbol{B}=B \boldsymbol{b}$, pointing in the $x_{3}$-direction. We assume the $E \times B$ velocity $\boldsymbol{u}$ to be orientated in direction $\boldsymbol{e}_{1}$, with amplitude linear in $x_{2}$, such that

$$
\boldsymbol{u}(\boldsymbol{x})=u_{1,2} x_{2} e_{1}
$$

corresponding to an electric field $\boldsymbol{E}=E_{2}\left(x_{2}\right) \boldsymbol{e}_{2}=u_{1,2} B x_{2} \boldsymbol{e}_{2}$ (cf. Figure 1). Here, we denote by $u_{1,2} \equiv \partial u_{1} / \partial x_{2}$ the partial derivative of the $x_{1}$-component of $\boldsymbol{u}$.

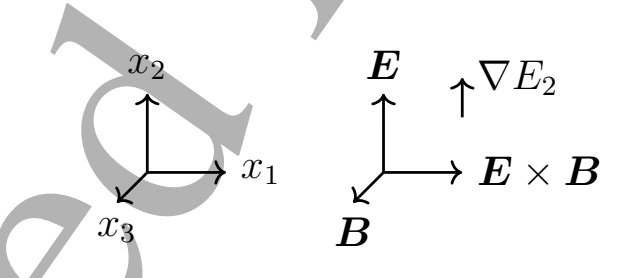

Figure 1: Assumed slab geometry.

\subsubsection{Particle trajectory}

We first derive the motion of a charged particle with mass $m$, charge $q$ as predicted by guiding-centre theory. We find

$$
\begin{aligned}
\boldsymbol{\rho}_{1} \cdot \boldsymbol{b} & =0, \\
\frac{1}{2 \mu} g_{\mu} & =-\frac{1}{2 \Omega} \boldsymbol{b} \cdot \nabla \times \boldsymbol{u}+\frac{1}{4 \Omega}(\widehat{\boldsymbol{\rho}} \widehat{\perp}+\widehat{\perp} \widehat{\boldsymbol{\rho}}): \nabla \boldsymbol{u}, \\
g_{\zeta} & =-\frac{1}{4 \Omega}(\widehat{\boldsymbol{\rho}} \widehat{\boldsymbol{\rho}}-\widehat{\perp} \widehat{\perp}): \nabla \boldsymbol{u}
\end{aligned}
$$


where $\boldsymbol{b} \cdot \nabla \times \boldsymbol{u}=-u_{1,2}$, and $\nabla \boldsymbol{u}=u_{1,2} \boldsymbol{e}_{2} \boldsymbol{e}_{1}$. Also $\Omega \equiv q B / m$ is the gyrofrequency. So that

$$
\begin{aligned}
& (\widehat{\boldsymbol{\rho}} \hat{\perp}+\widehat{\perp} \widehat{\boldsymbol{\rho}}): \nabla \boldsymbol{u}=u_{1,2}\left[\sin ^{2}(\zeta)-\cos ^{2}(\zeta)\right] \\
& (\widehat{\boldsymbol{\rho}} \widehat{\boldsymbol{\rho}}-\widehat{\perp} \widehat{\perp}): \nabla \boldsymbol{u}=u_{1,2}[-2 \cos (\zeta) \sin (\zeta)] .
\end{aligned}
$$

We then find from equations (5)-(7): $\boldsymbol{\rho}_{0}=\rho_{0}\left(\cos (\zeta) \boldsymbol{e}_{1}-\sin (\zeta) \boldsymbol{e}_{2}\right)$, and

$$
\boldsymbol{\rho}_{1}=\frac{3 \rho_{0} u_{1,2}}{4 \Omega} \boldsymbol{\rho}_{\mathbf{0}}+\frac{\rho_{0} u_{1,2}}{2 \Omega} \sin (\zeta) \boldsymbol{e}_{2} .
$$

Introducing $a \equiv \rho_{0}\left(1+\frac{3 u_{1,2}}{4 \Omega}\right)$, we can finally write

$$
\boldsymbol{\rho} \approx \boldsymbol{\rho}_{0}+\epsilon_{B} \boldsymbol{\rho}_{1}=a \cos (\zeta) \boldsymbol{e}_{1}-a\left(\frac{\Omega-\epsilon_{B} \frac{1}{2} u_{1,2}}{\Omega}\right) \sin (\zeta) \boldsymbol{e}_{2}
$$

Observing also that $\dot{\boldsymbol{X}}=\boldsymbol{u}(\boldsymbol{X})$ with $\boldsymbol{X}(t=0)=X_{1}^{(0)} \boldsymbol{e}_{1}+X_{2}^{(0)} \boldsymbol{e}_{2}$ implies $\boldsymbol{X}=\boldsymbol{X}(t=$ $0)+u_{1,2} X_{2}^{(0)} t \boldsymbol{e}_{1}$, it follows from $\boldsymbol{x}=\boldsymbol{X}+\boldsymbol{\rho}$ that

$$
\left\{\begin{array}{l}
x_{1}(t)=X_{1}^{(0)}+u_{1,2} X_{2}^{(0)} t+a \cos (\zeta(t)), \\
x_{2}(t)=X_{2}^{(0)}-a\left(\frac{\Omega-\epsilon_{B} \frac{1}{2} u_{1,2}}{\Omega}\right) \sin (\zeta(t)) .
\end{array}\right.
$$

In addition, we note that the guiding-centre equations of motion yield

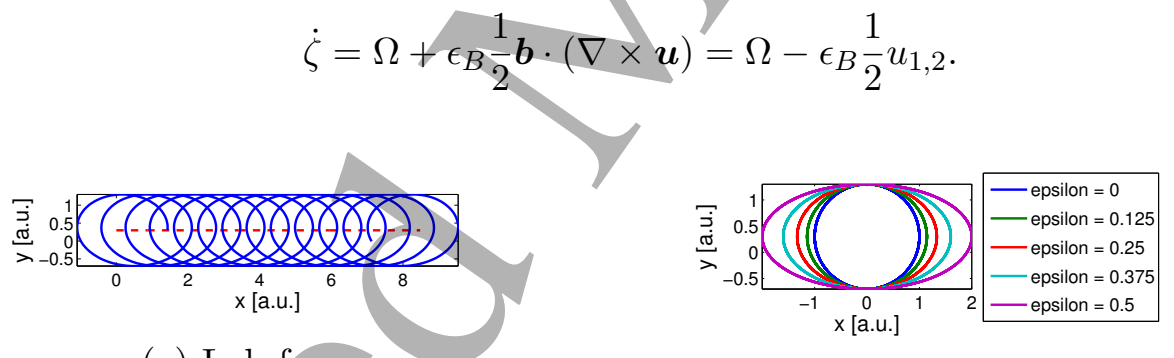

(a) Lab frame

(b) $E \times B$ frame

Figure 2: Particle motion in slab geometry with $\nabla E_{2} \neq 0$ : motion in lab frame with $E \times B$ drift clearly visible (left). Motion in frame co-moving with the guiding-centre (right) clearly shows deviation from circular motion due to $\nabla E_{2}$. In this figure, epsilon stands for $\epsilon \equiv$ $\left|\nabla \boldsymbol{u}_{E}\right| / \Omega_{c}=\left|\nabla E_{2}\right| / B \Omega_{c}$.

We can compare these guiding-centre results with the results obtained from the Lorentzian equations of motion [5]. According to [5, equation (20)], the general solution of the resulting Lorentzian equations of motion is given by

$$
\left\{\begin{array}{l}
x_{1}(t)=x_{1}^{(0)}+u_{1,2} x_{2}^{(0)} t+a \cos \left(\sqrt{\Omega\left(\Omega-\epsilon_{B} u_{1,2}\right)} t+\alpha\right) \\
x_{2}(t)=x_{2}^{(0)}-a\left(\frac{\Omega-\epsilon_{B} u_{1,2}}{\Omega}\right)^{1 / 2} \sin \left(\sqrt{\Omega\left(\Omega-\epsilon_{B} u_{1,2}\right)} t+\alpha\right) .
\end{array}\right.
$$


1

2

3

4

5

6

7

8

where $x_{1}^{(0)}, x_{2}^{(0)}, a, \alpha$ are constants to be determined from the initial conditions. By inspection of $(9)$ and $(11)$, we can immediately identify $\boldsymbol{x}^{(0)}=\boldsymbol{X}^{(0)}$, and we see that the leading-order approximation

$$
\zeta=\sqrt{\Omega\left(\Omega-\epsilon_{B} u_{1,2}\right)} t+\alpha \Rightarrow \dot{\zeta} \approx \Omega-\epsilon_{B} \frac{1}{2} u_{1,2}=\Omega-\epsilon_{B} \frac{1}{2} \boldsymbol{b} \cdot(\nabla \times \boldsymbol{u})
$$

is consistent with the guiding-centre picture (cp. equation $(10)$ ).
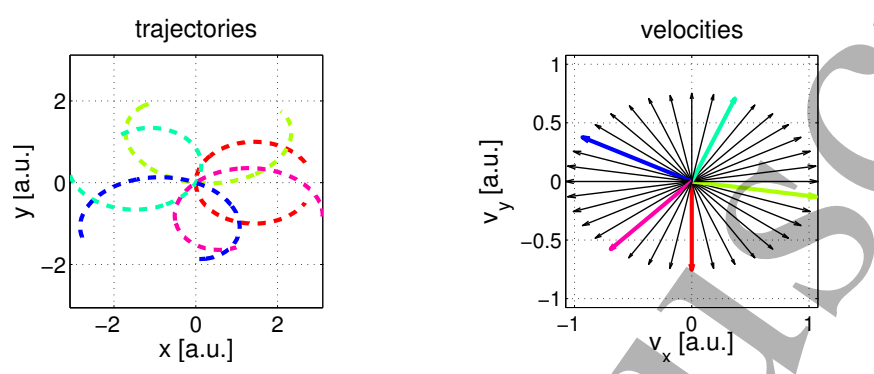

Figure 3: Trajectories intersecting $\boldsymbol{r}=0$ and corresponding anisotropic velocity distribution.

\subsubsection{Effects due to higher-order corrections}

The fact that the particle trajectory has an elliptic shape, rather than a circular one, turns out to cause off-diagonal terms in the pressure tensor. To see this, we fix a physical position $\boldsymbol{r}=0$ and consider all particle trajectories that intersect $\boldsymbol{r}$, as depicted in Figure 3. The fact that the trajectories are elliptical rather than circular translates into an anisotropy in the velocity distribution at $\boldsymbol{r}$ (cf. Figure 3 (right)).

The off-diagonal components $\boldsymbol{\Pi}_{12} \sim\left\langle v_{1} v_{2}\right\rangle, \boldsymbol{\Pi}_{11}-\boldsymbol{\Pi}_{22} \sim\left\langle v_{1}^{2}-v_{2}^{2}\right\rangle$ of the pressure tensor are non-zero because this anisotropy corresponds to correlations between $v_{1}, v_{2}$ as depicted in Figure 4 , leading to $\Pi \neq 0$. Here we have denoted the gyro-average by $\langle\ldots\rangle \equiv(2 \pi)^{-1} \int \mathrm{d} \zeta(\ldots)$.

Figure 4. Terms related to off-diagonal components $\boldsymbol{\Pi}_{12}$ and $\boldsymbol{\Pi}_{11}-\boldsymbol{\Pi}_{22}$ as function of gyroangle $\zeta$ at fixed $\boldsymbol{r}$ (solid), and their gyroaverages (dashed). Here: $v_{x} \equiv v_{1}, v_{y} \equiv v_{2}$. 


\subsection{General expression for pressure}

\subsubsection{Approach}

Let $\overline{\boldsymbol{u}}=\boldsymbol{u}+\epsilon_{B} \boldsymbol{u}_{*}+O\left(\epsilon_{B}^{2}\right)$ denote the (macroscopic) mass fluid velocity, including a leading-order term $\boldsymbol{u}$ with respect to which the guiding-centre equations are defined. The exact form of $\boldsymbol{u}$ in our context will be specified later on, for now we only require that the difference between $\boldsymbol{u}$ and $\overline{\boldsymbol{u}}$ is of higher order in $\epsilon_{B}$. We wish to evaluate the pressure tensor, retaining effects related to gradients in $\boldsymbol{u}$ to first order in $\epsilon_{B}$. The first step is to recognize that the distinction between $\boldsymbol{u}$ and $\overline{\boldsymbol{u}}$ will be unimportant for the evaluation of the pressure. The difference between these velocities, $\epsilon_{B} \boldsymbol{u}_{*}$, is a diamagnetic flow, which itself is of collective (distribution) origin. To see why the contribution due to $\epsilon_{B} \boldsymbol{u}_{*}$ is unimportant, let us first write the pressure tensor $\boldsymbol{P}$ in guiding-centre coordinates.

$$
\begin{aligned}
\boldsymbol{P}(\boldsymbol{r}) & =\int \mathrm{d}^{3} \boldsymbol{v} m[\boldsymbol{v}-\overline{\boldsymbol{u}}(\boldsymbol{r})][\boldsymbol{v}-\overline{\boldsymbol{u}}(\boldsymbol{r})] f(\boldsymbol{r}, \boldsymbol{v}) \\
& =\int \mathrm{d}^{6} \mathcal{Z}_{\mathrm{phys}} \delta(\boldsymbol{x}-\boldsymbol{r}) m[\boldsymbol{v}-\overline{\boldsymbol{u}}(\boldsymbol{r})][\boldsymbol{v}-\overline{\boldsymbol{u}}(\boldsymbol{r})] f\left(\mathcal{Z}_{\mathrm{phy}}\right) \\
& =\int \mathrm{d}^{6} \mathcal{Z}_{\mathrm{gc}} \delta(\boldsymbol{X}+\boldsymbol{\rho}-\boldsymbol{r}) m[\boldsymbol{v}-\overline{\boldsymbol{u}}(\boldsymbol{r})][\boldsymbol{v}-\overline{\boldsymbol{u}}(\boldsymbol{r})] F\left(\mathcal{Z}_{\mathrm{gc}}\right) .
\end{aligned}
$$

We have denoted $\mathcal{Z}_{\text {phy }}=(\boldsymbol{x}, \boldsymbol{v})$ the particle phase-space coordinates and $\mathcal{Z}_{\text {gc }}$ the guiding-centre phase-space coordinates, and $f, F$ are the distributions functions expressed in particle and guiding-centre coordinates, respectively.

Expanding now $\overline{\boldsymbol{u}}=\boldsymbol{u}+\epsilon_{B} \boldsymbol{u}_{*}$, and recognizing that the leading order particle velocity $\boldsymbol{v}=\boldsymbol{u}+w \boldsymbol{b}+\dot{\boldsymbol{\rho}}_{0}+O\left(\epsilon_{B}\right)$, we find

$$
\begin{aligned}
\boldsymbol{P}(\boldsymbol{r})= & \int \mathrm{d}^{6} \mathcal{Z}_{\mathrm{gc}} \delta\left(\boldsymbol{X}+\epsilon_{B} \boldsymbol{\rho}_{0}-\boldsymbol{r}\right) m[\boldsymbol{v}-\boldsymbol{u}(\boldsymbol{r})][\boldsymbol{v}-\boldsymbol{u}(\boldsymbol{r})] F\left(\mathcal{Z}_{\mathrm{gc}}\right) \\
& +\int \mathrm{d}^{6} \mathcal{Z}_{\mathrm{gc}} \delta\left(\boldsymbol{X}+\epsilon_{B} \boldsymbol{\rho}_{0}-\boldsymbol{r}\right) m[\boldsymbol{v}-\boldsymbol{u}(\boldsymbol{r})]\left[-\epsilon_{B} \boldsymbol{u}_{*}(\boldsymbol{r})\right] F\left(\mathcal{Z}_{\mathrm{gc}}\right)+(\mathrm{T})+O\left(\epsilon_{B}{ }^{2}\right) \\
= & \int \mathrm{d}^{6} \mathcal{Z}_{\mathrm{gc}} \delta\left(\boldsymbol{X}+\epsilon_{B} \boldsymbol{\rho}_{0}-\boldsymbol{r}\right) m[\boldsymbol{v}-\boldsymbol{u}(\boldsymbol{r})][\boldsymbol{v}-\boldsymbol{u}(\boldsymbol{r})] F\left(\mathcal{Z}_{\mathrm{gc}}\right) \\
& -\epsilon_{B} \boldsymbol{u}_{*}(\boldsymbol{r}) \int \mathrm{d}^{6} \mathcal{Z}_{\mathrm{gc}} \delta(\boldsymbol{X}-\boldsymbol{r}) m\left[w \boldsymbol{b}+\dot{\boldsymbol{\rho}}_{0}\right] F\left(\mathcal{Z}_{\mathrm{gc}}\right)+(\mathrm{T})+O\left(\epsilon_{B}{ }^{2}\right) .
\end{aligned}
$$

We denote by $(T)$ the transpose of the second term. Now, we find that the second term in the last equation is of order $\epsilon_{B}^{2}$, because

$$
\int \mathrm{d}^{3} \boldsymbol{v}_{\mathrm{gc}} m\left[w \boldsymbol{b}+\dot{\boldsymbol{\rho}}_{0}\right] F\left(\mathcal{Z}_{\mathrm{gc}}\right)=O\left(\epsilon_{B}\right)
$$

by our choice of parallel velocity variable $w$ as an (approximate) thermal fluctuation. We conclude that

$$
\boldsymbol{P}(\boldsymbol{r})=\int \mathrm{d}^{6} \mathcal{Z}_{\mathrm{gc}} \delta\left(\boldsymbol{X}+\epsilon_{B} \boldsymbol{\rho}_{0}-\boldsymbol{r}\right) m[\boldsymbol{v}-\boldsymbol{u}(\boldsymbol{r})][\boldsymbol{v}-\boldsymbol{u}(\boldsymbol{r})] F\left(\mathcal{Z}_{\mathrm{gc}}\right)+O\left(\epsilon_{B}{ }^{2}\right) .
$$

The goal of this section is to expand this expression for $\boldsymbol{P}$, retaining all corrections at order $\epsilon_{B}$. In guiding-centre coordinates $\mathcal{Z}_{\mathrm{gc}}$, we find

$$
\boldsymbol{v}=\dot{\boldsymbol{x}}=\dot{\boldsymbol{X}}+\dot{\rho} .
$$


The guiding centre velocity $\dot{\boldsymbol{X}}$ can be written in the form (cp. eq. (8))

$$
\dot{\boldsymbol{X}}=w \boldsymbol{b}(\boldsymbol{X})+\boldsymbol{u}(\boldsymbol{X})+\epsilon_{B} \boldsymbol{V}_{\mathrm{gc}}+O\left(\epsilon_{B}^{2}\right)
$$

It is important to note that $\boldsymbol{u}(\boldsymbol{r}) \neq \boldsymbol{u}(\boldsymbol{X})$. To the required accuracy,

$$
\dot{\boldsymbol{X}}-\boldsymbol{u}(\boldsymbol{r})=\dot{\boldsymbol{X}}-\boldsymbol{u}(\boldsymbol{X})-\epsilon_{B} \boldsymbol{\rho}_{0} \cdot \nabla \boldsymbol{u}(\boldsymbol{X})+O\left(\epsilon_{B}{ }^{2}\right)
$$

so that

$$
\boldsymbol{P}(\boldsymbol{r})=\int \mathrm{d}^{6} \mathcal{Z}_{\mathrm{gc}} \delta\left(\boldsymbol{X}+\epsilon_{B} \boldsymbol{\rho}_{0}-\boldsymbol{r}\right) m\left[w \boldsymbol{b}+\dot{\boldsymbol{\rho}}_{\mathrm{gc}}+\epsilon_{B} \boldsymbol{V}_{\mathrm{gc}}-\epsilon_{B} \boldsymbol{\rho}_{0} \cdot \nabla \boldsymbol{u}\right]^{\otimes 2} F\left(\mathcal{Z}_{\mathrm{gc}}\right)
$$

Expanding $\delta\left(\boldsymbol{X}+\epsilon_{B} \boldsymbol{\rho}_{0}-\boldsymbol{r}\right)=\left[1+\epsilon_{B} \boldsymbol{\rho}_{0} \cdot \nabla\right] \delta(\boldsymbol{X}-\boldsymbol{r})+O\left(\epsilon_{B}^{2}\right)$, integrating by parts, and neglecting terms at order $O\left(\epsilon_{B}^{2}\right)$ and higher throughout, we arrive at

$$
\begin{aligned}
\boldsymbol{P}(\boldsymbol{r})= & \int \mathrm{d}^{6} \mathcal{Z}_{\mathrm{gc}} \delta(\boldsymbol{X}-\boldsymbol{r}) m\left[w \boldsymbol{b}+\dot{\boldsymbol{\rho}}+\epsilon_{B} \boldsymbol{V}_{\mathrm{gc}}-\epsilon_{B} \boldsymbol{\rho}_{0} \cdot \nabla \boldsymbol{u}\right]^{\otimes 2} F \\
& -\nabla \cdot \int \mathrm{d}^{6} \mathcal{Z}_{\mathrm{gc}} \delta(\boldsymbol{X}-\boldsymbol{r}) m \boldsymbol{\rho}_{0}[w \boldsymbol{b}+\dot{\boldsymbol{\rho}}][w \boldsymbol{b}+\dot{\boldsymbol{\rho}}] F
\end{aligned}
$$

We will furthermore write the (canonical) phase-space volume in the form $\mathrm{d}^{6} \mathcal{Z}_{\mathrm{gc}}=$ $\mathrm{d} \boldsymbol{X} \mathrm{d} \boldsymbol{v}_{\text {gc }}$, where the guiding-centre velocity volume element is $\mathrm{d} \boldsymbol{v}_{\text {gc }} \equiv m^{-1} B_{\|}^{*} \mathrm{~d} w \mathrm{~d} \mu \mathrm{d} \zeta$ and $B_{\|}^{*} \equiv \boldsymbol{b} \cdot \nabla \times\left(\boldsymbol{A}+\epsilon_{B} \frac{m}{q} \boldsymbol{W}\right)$.

Let us note that both $\dot{\boldsymbol{\rho}} \equiv \dot{\boldsymbol{\rho}}^{(0)}+\epsilon_{B} \dot{\boldsymbol{\rho}}^{(1)}+O\left(\epsilon_{B}^{2}\right)$ and $\boldsymbol{\rho}_{0}$ are purely oscillatory in gyroangle. Expanding the above expression for $\boldsymbol{P}$ and taking into account that several terms gyro-average to zero, we/obtain

$$
\begin{aligned}
\boldsymbol{P}(\boldsymbol{r})= & \left(\int \mathrm{d}^{3} \boldsymbol{v}_{\mathrm{gc}} m w^{2} F\right) \boldsymbol{b} \boldsymbol{b}+\left(\int \mathrm{d}^{3} \boldsymbol{v}_{\mathrm{gc}} m \dot{\boldsymbol{\rho}}^{(0)} \dot{\boldsymbol{\rho}}^{(0)} F\right) \\
& +\epsilon_{B} \int \mathrm{d}^{3} \boldsymbol{v}_{\mathrm{gc}} m w\left[\boldsymbol{b} \boldsymbol{V}_{\mathrm{gc}}+\boldsymbol{V}_{\mathrm{gc}} \boldsymbol{b}\right] F \\
& +\epsilon_{B} \int \mathrm{d}^{3} \boldsymbol{v}_{\mathrm{gc}} m\left[\dot{\boldsymbol{\rho}}^{(0)} \dot{\boldsymbol{\rho}}^{(1)}+\dot{\boldsymbol{\rho}}^{(1)} \dot{\boldsymbol{\rho}}^{(0)}\right] F \\
& -\epsilon_{B} \int \mathrm{d}^{3} \boldsymbol{v}_{\mathrm{gc}} m\left[\dot{\boldsymbol{\rho}}^{(0)}\left(\boldsymbol{\rho}_{0} \cdot \nabla \boldsymbol{u}\right)+\left(\boldsymbol{\rho}_{0} \cdot \nabla \boldsymbol{u}\right) \dot{\boldsymbol{\rho}}^{(0)}\right] F \\
& -\epsilon_{B} \nabla \cdot \int \mathrm{d}^{3} \boldsymbol{v}_{\mathrm{gc}} m w \boldsymbol{\rho}_{0}\left[\boldsymbol{b} \dot{\boldsymbol{\rho}}^{(0)}+\dot{\boldsymbol{\rho}}^{(0)} \boldsymbol{b}\right] F .
\end{aligned}
$$

We remind the reader that in these expressions $F$ denotes the guiding-centre distribution, and that

$$
\mathrm{d}^{3} \boldsymbol{v}_{\mathrm{gc}}=m^{-1} B_{\|}^{*} \mathrm{~d} w \mathrm{~d} \mu \mathrm{d} \zeta .
$$

where $B_{\|}^{*}=B+\epsilon_{B} \frac{m}{q} \boldsymbol{b} \cdot \nabla \times \boldsymbol{W}$. We also note that we are not including $O\left(\epsilon_{B}^{2}\right)$-terms, so that we may use $\mathrm{d}^{3} \boldsymbol{v}_{\text {gc }} \approx m^{-1} B \mathrm{~d} w \mathrm{~d} \mu \mathrm{d} \zeta$ to calculate the first-order correction terms (13)-16. 


\subsubsection{Leading-order expression}

Observing that

$$
\dot{\boldsymbol{\rho}}^{(0)}=\Omega \partial_{\zeta} \boldsymbol{\rho}_{0}=\sqrt{2 \mu B / m} \hat{\perp} \approx \boldsymbol{v}_{\perp}
$$

we find that the gyroaverage $m\left\langle\dot{\boldsymbol{\rho}}^{(0)} \dot{\boldsymbol{\rho}}^{(0)}\right\rangle=2 \mu B\langle\hat{\perp} \hat{\perp}\rangle=\mu B(\boldsymbol{I}-\boldsymbol{b} \boldsymbol{b})$, and the CGL form of the pressure tensor is recovered to lowest order

$$
\boldsymbol{P}=P_{\perp}(\boldsymbol{I}-\boldsymbol{b} \boldsymbol{b})+P_{\|} \boldsymbol{b} \boldsymbol{b}+O\left(\epsilon_{B}\right) .
$$

In these expressions, we define the perpendicular and parallel guiding-centre pressure moments as

$$
P_{\perp}=\int \mathrm{d}^{3} \boldsymbol{v}_{\mathrm{gc}} \mu B F, \quad P_{\|}=\int \mathrm{d}^{3} \boldsymbol{v}_{\mathrm{gc}} m w^{2} F
$$

For future reference, we also define the guiding-centre parallel fluxès of parallel heat $q_{\|}^{\|}$ and transverse heat $q_{\|}^{\perp}$ as follows:

$$
q_{\|}^{\|}=\int \mathrm{d}^{3} \boldsymbol{v}_{\mathrm{gc}} m w^{3} F, \quad q_{\|}^{\perp}=\int \mathrm{d}^{3} \boldsymbol{v}_{\mathrm{gc}} w \mu B F .
$$

\subsubsection{Higher-order correction}

Detailed calculations for the higher-order terms have been included in the appendix. Here we only cite the final results. It is convenient to decompose the pressure tensor as follows:

$$
\boldsymbol{P}=\boldsymbol{P}^{\mathrm{CGL}}+\Pi_{\delta}\left(\boldsymbol{e}_{2} \boldsymbol{e}_{2}-\boldsymbol{e}_{1} \boldsymbol{e}_{1}\right)+\Pi_{12}\left(\boldsymbol{e}_{1} \boldsymbol{e}_{2}+\boldsymbol{e}_{2} \boldsymbol{e}_{1}\right)+\boldsymbol{b} \otimes \boldsymbol{\Pi}_{\boldsymbol{b} \perp}+\boldsymbol{\Pi}_{\perp \boldsymbol{b}} \otimes \boldsymbol{b},
$$

where the last two contributions are defined as

$$
\boldsymbol{\Pi}_{\boldsymbol{b} \perp} \equiv \boldsymbol{b} \cdot \boldsymbol{P} \cdot(\boldsymbol{I} \rightarrow \boldsymbol{b} \boldsymbol{b}), \quad \boldsymbol{\Pi}_{\perp \boldsymbol{b}} \equiv(\boldsymbol{I}-\boldsymbol{b} \boldsymbol{b}) \cdot \boldsymbol{P} \cdot \boldsymbol{b},
$$

and we note that by symmetry $\boldsymbol{\Pi}_{\boldsymbol{b} \perp}=\boldsymbol{\Pi}_{\perp \boldsymbol{b}}$.

Correction (I) (eq. (13)): From perpendicular Ohm's law, we have $[\mathrm{d} \boldsymbol{b} / \mathrm{d} t]_{\perp} \approx$ $[\boldsymbol{b} \cdot \nabla \boldsymbol{u}]_{\perp}$. This term therefore gives the following non-zero contributions

$$
\begin{aligned}
\Pi_{\boldsymbol{b} \perp} & =\boldsymbol{b} \otimes\left\{\frac{1}{\Omega} \boldsymbol{b} \times\left[2 P_{\|} \boldsymbol{b} \cdot \nabla \boldsymbol{u}+q_{\|}^{\perp} \nabla B+2 q_{\|}^{\|} \boldsymbol{\kappa}\right]\right\} \\
\Pi_{\delta} & =0 \\
\Pi_{12} & =0 .
\end{aligned}
$$

Correction (II) (eq. (14)): We find

$$
\begin{aligned}
\boldsymbol{\Pi}_{\boldsymbol{b} \perp} & =\boldsymbol{b} \otimes\left\{\frac{1}{\Omega} \boldsymbol{b} \times\left[-q_{\|}^{\perp} \boldsymbol{\kappa}-P_{\perp} \boldsymbol{b} \cdot \nabla \boldsymbol{u}\right]\right\} \\
\Pi_{\delta} & =\frac{1}{2 \Omega} \boldsymbol{I}_{\gamma}:\left[P_{\perp} \nabla \boldsymbol{u}+q_{\|}^{\perp} \nabla \boldsymbol{b}\right] \\
\Pi_{12} & =\frac{1}{2 \Omega} \boldsymbol{I}_{\delta}:\left[P_{\perp} \nabla \boldsymbol{u}+q_{\|}^{\perp} \nabla \boldsymbol{b}\right] .
\end{aligned}
$$


1

2

3

4

5

6

7

8

Correction (III): (eq. (15))

$$
\begin{aligned}
\boldsymbol{\Pi}_{\boldsymbol{b} \perp} & =\boldsymbol{b} \otimes\left\{\frac{1}{\Omega} \boldsymbol{b} \times\left[P_{\perp} \nabla \boldsymbol{u} \cdot \boldsymbol{b}\right]\right\} \\
\Pi_{\delta} & =\frac{1}{\Omega} \boldsymbol{I}_{\gamma}:\left[P_{\perp} \nabla \boldsymbol{u}\right] \\
\Pi_{12} & =\frac{1}{\Omega} \boldsymbol{I}_{\delta}:\left[P_{\perp} \nabla \boldsymbol{u}\right] .
\end{aligned}
$$

Correction (IV): (eq. 16)

$$
\begin{aligned}
\boldsymbol{\Pi}_{\boldsymbol{b} \perp} & =\boldsymbol{b} \otimes\left\{\frac{1}{\Omega} \boldsymbol{b} \times\left[\nabla q_{\|}^{\perp}-q_{\|}^{\perp} \nabla B-q_{\|}^{\perp} \boldsymbol{\kappa}\right]\right\} \\
\Pi_{\delta} & =\frac{1}{\Omega} \boldsymbol{I}_{\gamma}:\left[q_{\|}^{\perp} \nabla b\right] \\
\Pi_{12} & =\frac{1}{\Omega} \boldsymbol{I}_{\delta}:\left[q_{\|}^{\perp} \nabla b\right] .
\end{aligned}
$$

Sum of all terms (I-IV): Based on the above expressions, we finally obtain:

$$
\begin{aligned}
& \boldsymbol{\Pi}_{\boldsymbol{b} \perp}=\boldsymbol{b} \otimes\left\{\frac{1}{\Omega} \boldsymbol{b}\right. \times\left[\left(2 P_{\|}-P_{\perp}\right) \boldsymbol{b} \cdot \nabla \boldsymbol{u}+P_{\perp}(\nabla \boldsymbol{u}) \cdot \boldsymbol{b}\right. \\
&\left.\left.+2\left(q_{\|}^{\|}-q_{||}^{\perp}\right) \boldsymbol{\kappa}+\nabla q_{\| \mid}^{\perp}\right]\right\}, \\
& \Pi_{\delta}= \frac{1}{2 \Omega} \boldsymbol{I}_{\gamma}:\left[P_{\perp} \nabla \boldsymbol{u}+q_{\|}^{\perp} \nabla b\right], \\
& \Pi_{12}=\frac{1}{2 \Omega} \boldsymbol{I}_{\delta}:\left[P_{\perp} \nabla \boldsymbol{u}+q_{\|}^{\perp} \nabla b\right] .
\end{aligned}
$$

In addition, using $\boldsymbol{\Pi}_{\perp \boldsymbol{b}}=\left(\boldsymbol{\Pi}_{\boldsymbol{b} \perp}\right)^{T}$, we have for completion,

$$
\begin{array}{r}
\boldsymbol{\Pi}_{\perp \boldsymbol{b}}=\left\{\frac{1}{\Omega} \boldsymbol{b} \times\left[\left(2 P_{\|}-P_{\perp}\right) \boldsymbol{b} \cdot \nabla \boldsymbol{u}+P_{\perp}(\nabla \boldsymbol{u}) \cdot \boldsymbol{b}\right.\right. \\
\left.\left.+2\left(q_{\|}^{\|}-q_{\|}^{\perp}\right) \boldsymbol{\kappa}+\nabla q_{\|}^{\perp}\right]\right\} \otimes \boldsymbol{b}
\end{array}
$$

This form of the gyroviscous tensor, as derived from guiding-centre theory, recovers MacMahon's result [8, eq. (11)-(13)]. Macmahon's result remains the most general expression for the gyroviscous tensor for collisionless dynamics, and under the present fast-dynamics ordering, with fluid velocity of the order of the ion thermal velocity $\boldsymbol{u} \sim v_{\mathrm{th}, i}$. Note that the more conventionally cited expression for the gyroviscous tensor due to Braginskii [22] is instead based on the assumption of fast dynamics and high collisionality, and hence may be appropriate if fluid equations with an ad hoc closure are discussed, but does not pertain in the present context. A thorough discussion and comparison of several alternate orderings for FLR corrected fluid equations has been given by Ramos [9, 10], and we refer the interested reader to that work for further information. For the present work, it is interesting to point out that those contributions to the gyroviscous tensor, which are proportional to gradients in $\boldsymbol{u}$, are the same for 
all the various orderings discussed in [10]. It is this contribution proportional to $\nabla \boldsymbol{u}$ which is most commonly considered, and which is responsible for the approximate gyroviscous cancellation. For a discussion of the derivation of the gyroviscous force from the gyroviscous tensor, and the approximate gyroviscous cancellation in the momentum equation that is implied by the gyroviscous force, we refer to [3, 4, 5, 6, 17, 10, 22. In particular, our derivation shows that the generalized guiding-centre theory developed in the present work is consistent with the inclusion of finite Larmor-radius effects (diamagnetic flows) in the momentum equation.

\section{A Kinetic-MHD model for strong flows}

\subsection{Nonlinear formulation including diamagnetic effects}

\subsubsection{Kinetic-MHD model}

Based on the above development of guiding-centre theory in terms of the MHD-like velocity $\boldsymbol{u}$, and the demonstration that this formulation is consistent with the gyroviscous cancellation, we can now re-write the exact momentum equation, obtained in the limit $m_{e} \rightarrow 0$ : the exact momentum equation in this limit is given in terms of the ion flow velocity (we assume a single ion species) $\overline{\boldsymbol{u}}=\boldsymbol{u}+\boldsymbol{u}_{*, i}+O\left(\epsilon_{B}^{2}\right)$ :

$$
m_{i} n\left(\frac{\partial \overline{\boldsymbol{u}}}{\partial t}+\overline{\boldsymbol{u}} \cdot \nabla \overline{\boldsymbol{u}}\right)+\nabla \cdot \boldsymbol{\Pi}_{i}=\boldsymbol{j} \times \boldsymbol{B}-\nabla \cdot \boldsymbol{P}^{\mathrm{CGL}} .
$$

While the derivation in the last section has been based on a guiding-centre calculation, the resulting expression for $\boldsymbol{\Pi}_{i}$ agrees with known results obtained from fluid theory. Therefore following [23, and employing the approximate gyro-viscous cancellation 3 we write

$$
m_{i} n\left(\frac{\partial \overline{\boldsymbol{u}}}{\partial t}+\overline{\boldsymbol{u}} \cdot \nabla \overline{\boldsymbol{u}}\right)+\nabla \cdot \boldsymbol{\Pi}_{i} \approx m_{i} n \frac{\mathrm{d} \boldsymbol{u}}{\mathrm{d} t}+\boldsymbol{u}_{*, i} \cdot \nabla \boldsymbol{u}_{\perp},
$$

with $\frac{\mathrm{d}}{\mathrm{d} t}=\partial_{t}+\boldsymbol{u} \cdot \nabla$ the convective derivative along $\boldsymbol{u}$. Thus, we find the following form of the momentum equation

$$
m_{i} n \frac{\mathrm{d} \boldsymbol{u}}{\mathrm{d} t}+\boldsymbol{u}_{*, i} \cdot \nabla \boldsymbol{u}_{\perp}=\boldsymbol{j} \times \boldsymbol{B}-\nabla \cdot \boldsymbol{P}^{\mathrm{CGL}}
$$

where

$$
\boldsymbol{u}_{*, i}=\frac{\boldsymbol{b} \times\left(\nabla \cdot \boldsymbol{P}_{i}^{\mathrm{CGL}}\right)}{Z e B}
$$

is the ion diamagnetic flow. In this last expression, we have allowed for a general Chew-Goldberger-Low form $\boldsymbol{P}^{\mathrm{CGL}}$, instead of the isotropic form $\boldsymbol{P}^{\mathrm{CGL}}=p \boldsymbol{I}$, which was assumed in the fluid treatment [23]. The density $n$ in $(20)$ satisfies the continuity equation

$$
\frac{\partial n}{\partial t}+\nabla \cdot(n \boldsymbol{u})=0
$$

${ }^{3}$ We employ the approximate form of the gyroviscous force at this point, rather than the exact expression as e.g. derived in [10], mainly for simplicity of the resulting equations. 
1

2

3

4

5

6

7

8

10

Assuming $\boldsymbol{E}_{\|} \approx 0$, Ohm's law can be written:

$$
\boldsymbol{E}+\boldsymbol{u} \times \boldsymbol{B}=0
$$

This form of Ohm's law then leads to the conventional ideal MHD result

$$
\frac{\partial \boldsymbol{B}}{\partial t}=\nabla \times(\boldsymbol{u} \times \boldsymbol{B}) .
$$

Finally, closure is provided by the solution of the collisionless guiding-centre Vlasov equation

$$
\partial_{t} F_{s, \mathrm{gc}}+\dot{\boldsymbol{X}} \cdot \nabla F_{s, \mathrm{gc}}+\dot{w} \frac{\partial F_{s, \mathrm{gc}}}{\partial w}=0
$$

for each species $s \in\{i, e\}$. We note that

$$
\dot{\boldsymbol{X}}=w \frac{\boldsymbol{B}^{*}}{B_{\|}^{*}}+\frac{\boldsymbol{E}^{*} \times \boldsymbol{b}}{B_{\|}}, \quad \dot{w}=\frac{q_{s}}{m_{s}} \frac{\boldsymbol{E}^{*} \cdot \boldsymbol{B}^{*}}{B B_{\|}^{*}},
$$

with $\boldsymbol{E}^{*} \equiv-\nabla H / q-\frac{\partial \boldsymbol{A}^{*}}{\partial t}, \boldsymbol{B}^{*} \equiv \nabla \times \boldsymbol{A}^{*}$ and $\boldsymbol{A}^{*} \equiv \boldsymbol{A}+\frac{m}{q} \boldsymbol{W}$, are derived from the guiding-centre Lagrangian $\mathcal{L}$ (cp. equation (3)). The pressure tensor $\boldsymbol{P}^{\mathrm{CGL}}$ is computed at leading order as

where

$$
\begin{aligned}
& \boldsymbol{P}^{\mathrm{CGL}}=\sum_{s} \boldsymbol{P}_{s}^{\mathrm{CGL}}, \\
& \boldsymbol{P}_{s}^{\mathrm{CGL}}=P_{s, \|} \boldsymbol{b} \boldsymbol{b}+P_{s, \perp}(\boldsymbol{I}-\boldsymbol{b} \boldsymbol{b}),
\end{aligned}
$$

$$
\begin{aligned}
P_{s, \|} & \equiv \int m_{s} w^{2} F_{s, \mathrm{gc}} \mathrm{d}^{3} \boldsymbol{v}_{\mathrm{gc}} \\
P_{s, \perp} & \equiv \int \mu B F_{s, \mathrm{gc}} \mathrm{d}^{3} \boldsymbol{v}_{\mathrm{gc}} .
\end{aligned}
$$

The resulting system of equations is written in term of the variables $\left(n, \boldsymbol{u}, \boldsymbol{B}, F_{s}\right)$, where $n$ is the ion number density, $\boldsymbol{u}$ is the leading-order fluid velocity, $\boldsymbol{B}$ is the total magnetic field, and $F_{s}$ denote the the guiding-centre distribution functions for each species $s$. The numerical solution of the non-linear kinetic-MHD system requires the evolution of $n$ according to equation (21), $\boldsymbol{u}$ is solved for from equation (20) and the solution of Ohm's law (22) is needed to advance $\boldsymbol{B}$. In addition, the guiding-centre distributions are evolved according to the collisionless guiding-centre Vlasov equation (23), from which the CGL-contribution to the pressure are obtained according to (25). The resulting system of equations can be used to study the non-linear dynamics of low-frequency modes for which diamagnetic effects are important, i.e. when $\omega \sim n \omega_{*, i}$. Both FLR, as well as particle wave interactions (precession resonance) are included in this non-linear model. 


\subsubsection{Discussion of the non-linear kinetic-MHD model}

The kinetic-MHD approach has a long history going back at least to the pioneering work of Rosenbluth and Rostocker [24]. In this section we would like to point out a subtlety that make this approach particularly suitable for the study of kinetic effects on macroscopic instabilities. To explain why, we start out by introducing the formal ordering of the terms appearing in the momentum equation 20 :

$$
m_{i} n \frac{\mathrm{d} \boldsymbol{u}}{\mathrm{d} t}+\epsilon_{B} \boldsymbol{u}_{*, i} \cdot \nabla \boldsymbol{u}_{\perp}=\epsilon_{B}^{-1} \boldsymbol{j} \times \boldsymbol{B}-\nabla \cdot \boldsymbol{P}^{\mathrm{CGL}}
$$

In order to obtain a consistent set of equations, we need to provide closure relations for $\boldsymbol{j}$ and $\boldsymbol{P}^{\mathrm{CGL}}$. The crucial point of kinetic-MHD is that it is beneficial not to compute the current density directly from the particle distribution function $f_{s}$ (cp. the classic work by Rosenbluth, Rostocker [24, p.25], or the book by Freidberg [25, p.391]). Indeed, if we were to write $\boldsymbol{j}=q_{s} \int \boldsymbol{v} f_{s} \mathrm{~d}^{3} \boldsymbol{v}$, we would require knowledge of $f_{s}$ including corrections at order $\epsilon_{B}{ }^{2}$, in order to compute $j$ to an order that is consistent with the other terms in (26). Instead, we express $\boldsymbol{j}$ in terms of $\boldsymbol{B}$ using Ampere's law $\nabla \times \boldsymbol{B}=\mu_{0} \boldsymbol{j}$, and the evolution of $\boldsymbol{B}$ is governed by equation $(22)$.

Thus, the only variable that requires kinetic closure is now the CGL-part of the pressure tensor $\boldsymbol{P}^{\mathrm{CGL}}$. And comparing with the ordered momentum equation (26), we find that the pressure can be consistently computed from knowledge of the distribution function $f_{s}$ including FLR correction to order $\epsilon_{B}$. In this way, the kinetic-MHD approach with pressure closure achieves an efficient use of kinetic information, in the sense that the guiding-centre distribution $F_{S}$, computed based on guiding-centre theory including all $O\left(\epsilon_{B}\right)$-corrections is sufficient. Higher-order corrections at $O\left(\epsilon_{B}{ }^{2}\right)$ are not required in a kinetic-MHD approach, whereas they would be required to compute all terms in the momentum equation (26) from a purely kinetic approach.

Previous versions of the kinetic-MHD model, as e.g. discussed in [25, Chap. 9.5], have focused on leading-order effects, for which only information about the zeroth-order distribution function is necessary. In this work, we also consider first-order corrections in the guiding-centre equations which lead to important particle drift effects, and which allow us to consistenly include the effects due to the diamagnetic drift velocity in the momentum equation (26). We emphasize that the above non-linear model is based on the MHD form of Ohm's law (also parallel to the field lines), and hence does not include kinetic effects due to $\boldsymbol{E}_{\|} \neq 0$. In sections 4.3 and 4.4 below, we will instead consider a linearized kinetic-MHD model where the effects due to $\boldsymbol{E}_{\|} \neq 0$ are included.

In closing our discussion of the non-linear kinetic-MHD model, we remark that a similar pressure-coupling approach is for example followed in the numerical code XTOR-K, which is currently under development. XTOR-K is a extension of the twofluid code XTOR-2F [17, 16]. In XTOR-K, kinetic closure is achieved by computing the particle distribution function $f_{s}$, based on direct solution of the Vlasov equation, rather than a set of reduced guiding-centre equations. Numerically, this puts more stringent demands on the maximum allowed time-step size, since the fast gyro-motion of particles needs to be resolved in XTOR-K. In contrast the fastest motion in the guiding-centre description is on the order of the bounce-frequency of particles. The model proposed in this work could thus serve as a computationally less demanding alternative to provide kinetic closure for XTOR-K, in the collisionless limit. Similarly to our non-linear model, XTOR-K also does not determine $\boldsymbol{E}_{\|}$from a quasi-neutrality relation. 
In comparison to non-linear, global gyrokinetic codes [26, 27], we expect that the kinetic-MHD system might be less computationally expensive to solve in practice, due to the fact that the considered modes are long-wavelength, in contrast to the small-scale turbulence considered in gyrokinetics. Hence, we would expect such modes to require less spatial resolution. In addition, the kinetic-MHD treatment requires no (non-local) gyroaveraging operations to be carried out, and thus fewer operations per time-step.

While the guiding-centre equations which we used to derive the expression for the gyroviscous tensor (17) have naturally led to a non-linear kinetic-MHD model, the main goal of the present work is the derivation of a linear model, allowing for a nonzero parallel electric field. The following sections are devoted to the derivation of such a linear model.

\subsection{Equilibrium}

Disclaimer: In the following, we will consistently write fields as a sum of equilibrium and perturbed parts. Perturbed quantities will be denoted with a $\delta$. To ease notation, we will however not indicate all equilibrium quantities with a subscript 0 , e.g. we shall replace $\boldsymbol{B}(\boldsymbol{x}, t) \rightarrow \boldsymbol{B}(\boldsymbol{x})+\delta \boldsymbol{B}(\boldsymbol{x}, t)$. Unless otherwise specified, henceforth any quantity without a $\delta$ will refer to an equilibrium quantity.

Before deriving equations to study the linear dynamics, we briefly discuss the magnetohydrodynamic equilibrium from a kinetic point of view. We will assume for simplicity that the equilibrium is isotropic. So that the CGL pressure tensor becomes a scalar. Note though that the lowest order perturbed pressure is not isotropic. Following [19], we will assume an axisymmetric equilibrium with nested flux surfaces, so that we can choose flux coordinates $(\psi, \theta, \phi)$ with $\psi$ the poloidal flux variable, $\theta$ the poloidal angle and $\phi$ the toroidal angle. We furthermore assume the equilibrium flow, defining the co-moving frame, to be purely toroidal and given by

$$
\boldsymbol{u}_{0}=\Omega(\psi) R^{2} \nabla \phi
$$

where $\Omega(\psi)$ is the leading-order toroidal rotation frequency and $R=R(\psi, \theta)$ is the major radius. This is consistent with lowest-order kinetic theory for all collisionalities in an axisymmetric plasma [28]. We remind the reader that the rotation frequency is related to the leading-order potential $\Phi_{0}(\psi)$ by $\Omega=-\mathrm{d} \Phi_{0} / \mathrm{d} \psi[19]$.

Guiding-centre equations allowing for strong toroidal flows have been developed in [19], where the following form of the equilibrium guiding-centre Lagrangian was derived:

$$
\begin{aligned}
& \Gamma_{0}=[q \boldsymbol{A}+m \boldsymbol{W}] \cdot \mathrm{d} \boldsymbol{X}+\frac{m}{q} \mu \mathrm{d} \zeta-H_{0} \mathrm{~d} t \\
& H_{0}=\frac{1}{2} m \boldsymbol{W}^{2}+\mu B+q \Phi_{0}+q \Phi_{1},
\end{aligned}
$$

where $\boldsymbol{W}=\boldsymbol{u}_{0}+w \boldsymbol{b}, \Phi_{0}$ is the leading-order electrostatic potential, $\boldsymbol{A}$ denotes the equilibrium vector potential, and $\boldsymbol{b}=\boldsymbol{B} / B$ with $\boldsymbol{B}=\nabla \phi \times \nabla \psi+F(\psi) \nabla \phi$ the magnetic field. We have allowed for a higher-order electrostatic correction $\Phi_{1}$, which is induced by centrifugal effects and which will be computed from quasi-neutrality below. It is imposed that the flux surface average of $\Phi_{1}$ vanish. We will denote the total electrostatic potential by $\Phi=\Phi_{0}+\Phi_{1}$. As guiding-centre variables, we take the guiding-centre 
position $\boldsymbol{X}$, the guiding-centre parallel velocity $w$ measured in a frame moving with $\boldsymbol{u}_{0}$, the magnetic moment $\mu$ suitably defined in the moving frame [19], and the gyroangle $\zeta$. We point out that 27) can be viewed as a special case of the non-linear guiding-centre Lagrangian (3) when the fields are at equilibrium.

Since the equilibrium is assumed axisymmetric and stationary, the coefficients of the Lagrangian do not explicitly depend on toroidal angle $\phi$ and time $t$, nor are they dependent on the gyroangle $\zeta$. Correspondingly, we have following the three constant of equilibrium guiding-centre motion: toroidal momentum $P_{\phi} \equiv q A_{\phi}+m W_{\phi}$, total energy $E=\frac{1}{2} m W^{2}+\mu B+q \Phi$ and magnetic moment $\mu$.

The equilibrium Hamiltonian $H_{0}$ corresponds to the energy $E$ as measured in the lab frame, and contains the formally large contribution $q \Phi_{0} \sim \epsilon_{B}^{-1} \frac{1}{2} m W^{2}$. It turns out that it will be useful to introduce a new constant of motion, $\mathcal{E}$, which does not contain such formally large terms. To this end and following Brizard [19], we define a pseudo-radial variable $\psi^{*}$ via the toroidal momentum $P_{\phi}$ :

$$
\psi^{*} \equiv-P_{\phi} / q=\psi-\frac{m}{q} W_{\phi}=\psi+\Delta \psi
$$

where $\Delta \psi=-\frac{m}{q} W_{\phi}$ is the banana width in units of $\psi$. Clearly, $\psi^{*}$ is a constant of motion in axisymmetric equilibria, so that we can define a new constant of motion as the combination $\mathcal{E} \equiv E-q \Phi_{0}\left(\psi^{*}\right)$. Expanding

$$
\begin{aligned}
\Phi_{0}\left(\psi^{*}\right) & \approx \Phi_{0}(\psi)-\frac{m}{q} W_{\phi} \frac{\mathrm{d} \Phi_{0}}{\mathrm{~d} \psi} \\
& =\Phi_{0}(\psi)+\frac{m}{q} \boldsymbol{W} \cdot \boldsymbol{u}_{0}
\end{aligned}
$$

and $\boldsymbol{W}=w \boldsymbol{b}+\boldsymbol{u}_{0}$, then including order $\Delta \psi$ corrections, we can write

$$
\begin{aligned}
\mathcal{E} & =E-q \Phi_{0}\left(\psi^{*}\right) \\
& \approx\left[\frac{1}{2} m w^{2}+m w \boldsymbol{b} \cdot \boldsymbol{u}_{0}+\frac{1}{2} m \boldsymbol{u}_{0}^{2}+q \Phi(\psi)\right] \\
& -q\left[\Phi_{0}(\psi)+\frac{m}{q}\left(w \boldsymbol{b}+\boldsymbol{u}_{0}\right) \cdot \boldsymbol{u}_{0}\right] \\
& =\frac{1}{2} m w^{2}+\mu B+q \Phi_{1}-\frac{1}{2} m \boldsymbol{u}_{0}^{2} .
\end{aligned}
$$

Thus, $\mathcal{E}$ corresponds to the energy measured in the rotating frame. Under collisionless dynamics, the equilibrium distribution function $F_{0}=F_{0}\left(P_{\phi}, E, \mu\right)$ of a given species must depend on the constants of motion, only. Instead of $P_{\phi}$ and $E$, we can equivalently use the constants of motion $\psi^{*}$ and $\mathcal{E}$. For thermal species $(s=i, e)$, we shall impose $F_{0}=F_{0}\left(\psi^{*}, \mathcal{E}\right)$ to be Máxwellian in $\mathcal{E}$, so that

$$
F_{0, s}\left(\psi^{*}, \mathcal{E}\right) \equiv \frac{N_{s}\left(\psi^{*}\right)}{\left(2 \pi T_{s}\left(\psi^{*}\right) / m_{s}\right)^{3 / 2}} \exp \left(-\mathcal{E} / T_{s}\left(\psi^{*}\right)\right)
$$

with $N_{s}(\psi), T_{s}(\psi)$ given profiles. Notice that this equilibrium distribution is isotropic $p_{\|}=p_{\perp}=p$. That the temperature $T_{s}(\psi)$ is a flux function is consistent with FokkerPlanck equation solutions of arbitrary collisionalities [28]. Thermal gradients cannot build up along magnetic field lines, where particles can freely stream. 
We next show that this natural choice for $F_{0, s}$ can be used to recover the ideal MHD equilibrium relations. The physical ion and electron densities are given by

$$
\begin{aligned}
n_{s}(\psi, \theta) & =\int \mathrm{d}^{3} \boldsymbol{v} F_{0, s} \\
& \approx N_{s}(\psi) \exp \left(\frac{-q \Phi_{1}+\frac{1}{2} m R^{2} \Omega^{2}}{T_{s}(\psi)}\right) .
\end{aligned}
$$

Assuming quasi-neutrality between electrons and ions, $n_{e}=Z n_{i}$, and neglecting centrifugal effects for electrons $\left(m_{e} \ll m_{i}\right)$, we obtain

$$
q \Phi_{1}=\frac{m_{i} \Omega^{2}}{2\left(1+T_{i} / T_{e}\right)}\left(R^{2}-\left\langle\left\langle R^{2}\right\rangle\right\rangle\right)
$$

where $\langle\langle\ldots\rangle\rangle$ denotes flux-surface averaging. Assuming in addition that $T_{i}=T_{e}$, we find that the mass density and pressure $p=p_{i}+p_{e}$ are poloidally dependent and of the form

We can now write

$$
\begin{aligned}
& \rho(\psi, \theta) \approx \bar{\rho}(\psi) \exp \left(\frac{m_{i} R^{2} \Omega^{2}}{4 T(\psi)}\right), \\
& p(\psi, \theta) \approx \bar{p}(\psi) \exp \left(\frac{m_{i} R^{2} \Omega^{2}}{4 T(\psi)}\right) .
\end{aligned}
$$

$$
\nabla p=\left.\frac{\partial p}{\partial \psi}\right|_{R} \nabla \psi+\left.\frac{\partial p}{\partial R}\right|_{\psi} \nabla R
$$

and recall that $\boldsymbol{u}_{0} \cdot \nabla \boldsymbol{u}_{0}=-\Omega^{2} R \nabla R$. From the parallel projection of equilibrium force balance, $\boldsymbol{b} \cdot\left(\rho \boldsymbol{u}_{0} \cdot \nabla \boldsymbol{u}_{0}+\nabla p\right)=0$, we then find the following relationship between $\rho, p, T$ :

$$
\rho=\frac{m_{i} p}{2 T} \Longleftrightarrow p=2 \rho T / m_{i}
$$

Note that $\rho=m_{i} n_{i}$ and $p=p_{i}+p_{e}=2 p_{i}$, so that the above is equivalent to $2 p_{i}=2 n_{i} T$. Recalling that $p=2 p_{i}$ and $n=n_{e}+n_{i}=2 n_{i}$, we arrive at the equation of state for an ideal gas $p=n T$.

Radial force balance leads to the conventional form of the Grad-Shafranov equation for toroidally rotating plasmas

$$
\Delta^{*} \psi+\left.\mu_{0} R^{2} \frac{\partial p}{\partial \psi}\right|_{R}+\frac{1}{2} \frac{\mathrm{d} F^{2}}{\mathrm{~d} \psi}=0, \quad \Delta^{*} \psi \equiv R \nabla \cdot\left(R^{-1} \nabla \psi\right)
$$

where we recall that the magnetic field is represented as $\boldsymbol{B}=\nabla \phi \times \nabla \psi+F(\psi) \nabla \phi$ and $\phi$ is the geometric toroidal angle. We have thus shown that our assumed Maxwellian form of the distribution function is consistent with ideal MHD equilibria. Note that a similar result has been obtained in [29]. In the present work, we have shown that the derivation of [29] is consistent with the guiding-centre equations in a co-moving frame (cf. section 2). 


\subsection{Linear Perturbation}

Next, we wish to derive suitable equations for the linear kinetic response. The physical parameters to consider are $\epsilon_{B} \sim \rho_{i} / L_{B}$ with $\rho_{i}$ the thermal ion Larmor radius and $L_{B}$ the background equilibrium length scale, $\delta \sim \delta B / B$ the amplitude of the perturbations and $k_{\perp} \rho_{i}$, with $k_{\perp}$ the perpendicular wave vector of the perturbed quantities. The validity of the guiding-centre transformation requires that $\epsilon_{B} \ll 1$. The study of linear dynamics imposes $\delta \ll 1$. Different forms of the linearized kinetic equation can then be derived assuming $k_{\perp} \rho_{i} \ll 1$ (guiding-centre approach), or allowing for $k_{\perp} \rho_{i} \lesssim 1$ (gyrokinetic approach). Clearly, for the study of the linearized dynamics, the gyrokinetic approach is the more general one, motivating us to follow that approach in the following.

\subsubsection{Gyrokinetic transformation}

We shall initially allow for $k_{\perp} \rho_{i} \sim 1$, before discussing the long-wavelength limit $k_{\perp} \rho_{i} \ll$ 1 in section 4.3.4. In section 4.3.5. we show that the results obtained from gyrokinetic theory in the long wavelength limit are consistent with the linearization of the guidingcentre equations of section 2 .

The gyrokinetic approach, as developed in [30, and references therein], is a twostep process: In a first step, a near-identity transformation is carried out to pass from particle coordinates $\mathcal{Z}_{\text {phy }}$ to guiding-centre coordinates $\mathcal{Z}_{\mathrm{gc}}$, defined with respect to the equilibrium fields. In a second step, perturbations are introduced. To obtain a reduced set of kinetic equations taking into account both background inhomogeneities as well as the perturbations, a second transformation to gyrocentre coordinates $\mathcal{Z}_{\text {gy }}$ is carried out (cf. Figure 5).

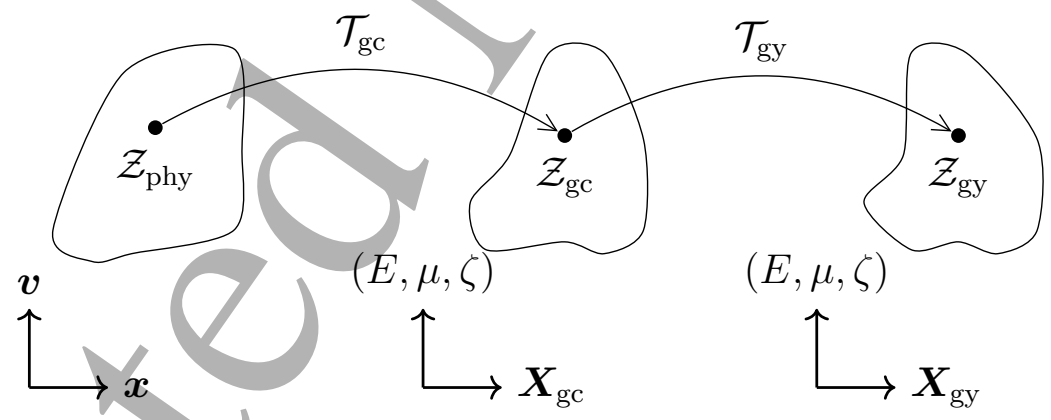

Figure 5: Two-step transformation from particle to gyro-centre coordinates.

After the guiding-centre transformation with respect to the equilibrium fields, explained in detail in [19], the Lagrangian in guiding-centre coordinates now takes the form $\Gamma=\Gamma_{0}+\Gamma_{1}$ with $\Gamma_{0}$ given by equation (27), and the perturbed Lagrangian,

$$
\Gamma_{1}=q \delta \boldsymbol{A}(\boldsymbol{X}+\boldsymbol{\rho}) \cdot \mathrm{d}[\boldsymbol{X}+\boldsymbol{\rho}]-q \delta \phi(\boldsymbol{X}+\boldsymbol{\rho}) \mathrm{d} t .
$$

We denote by $\boldsymbol{\rho}$ the guiding-centre displacement, written in guiding-centre coordinates, such that the particle position $\boldsymbol{x}$ is related to the guiding-center position $\boldsymbol{X}$ by $\boldsymbol{x}=$ $\boldsymbol{X}+\boldsymbol{\rho}$. Clearly, the perturbed Lagrangian $\Gamma_{1}$ is gyro-angle dependent, because of the 
gyro-angle dependent terms in $\boldsymbol{\rho}$. To remove this gyroangle-dependence we carry out a near identity transformation to gyrocentre coordinates induced by the phase-space vector field $\overline{\boldsymbol{G}}_{1}$. While the final result has previously been obtained in [19], we here supply a detailed discussion of the derivation. Following [30], the components of the perturbed Lagrangian in gyrocentre coordinates are related to the components of $\overline{\boldsymbol{G}}_{1}$ by

$$
\bar{G}_{1}^{a}=\left\{\bar{S}_{1}, \mathcal{Z}^{a}\right\}_{0}+\left(\Gamma_{1 b}-\bar{\Gamma}_{1 b}\right) J_{0}^{a b}
$$

where $\bar{S}_{1}$ is a gauge function, solving the equation

$$
\frac{\mathrm{d}_{0} \bar{S}_{1}}{\mathrm{~d} t}=\left(\widetilde{H_{1}-\bar{H}_{1}}\right)-\left(\widetilde{\Gamma_{1 a}-\bar{\Gamma}_{1 a}}\right) \dot{\mathcal{Z}}_{0}^{a}
$$

and $\widetilde{(\ldots)}$ denotes the gyro-angle oscillatory part of a quantity, and $\dot{\mathcal{Z}}_{0}^{a}=\left\{\mathcal{Z}^{a}, H_{0}\right\}$ is the time derivative along an unperturbed orbit. We shall choose a Hamiltonian representation, meaning that only perturbed terms in the Hamiltonian are present, i.e. we choose $\bar{\Gamma}_{1}=(\ldots) \mathrm{d} t$, with vanishing $\mathrm{d} \boldsymbol{X}$-components. It then follows that

$$
\left.\frac{\mathrm{d}_{0} \bar{S}_{1}}{\mathrm{~d} t}=q \widetilde{\delta_{*} \phi}-q\left[\delta_{*} \boldsymbol{A} \cdot \widetilde{\boldsymbol{X}}+\dot{\boldsymbol{\rho}}\right]\right],
$$

where we have introduced $\delta_{*} \phi \equiv \delta \phi(\boldsymbol{X}+\boldsymbol{\rho})$, and $\delta_{*} \boldsymbol{A} \equiv \delta \boldsymbol{A}(\boldsymbol{X}+\boldsymbol{\rho})$, following the notation in [19].

With this choice of $\overline{\boldsymbol{G}}_{1}$ and $\bar{S}_{1}$, the perturbed Hamiltonian in gyrocentre coordinates is given by

$$
\begin{aligned}
\bar{H}_{1} & =\left\langle H_{1}\right\rangle-\left(\Gamma_{1 a}-\bar{\Gamma}_{1 a}\right) \dot{\mathcal{Z}}_{0}^{a}-\left\{\bar{S}_{1}, H_{0}\right\}_{0} \\
& =q\left\langle\delta_{*} \phi\right\rangle-q\left\langle\delta_{*} \boldsymbol{A} \cdot[\dot{\boldsymbol{X}}+\dot{\boldsymbol{\rho}}]\right\rangle .
\end{aligned}
$$

where $\langle\ldots\rangle$ denotes gyro-averaging.

\subsubsection{Collisionless linear gyro-kinetic equation}

In Hamiltonian gyrocentre coordinates, the perturbation only affects the Hamiltonian part, so that the Poisson bracket (which is determined by the symplectic part of the Lagrangian) is formally the same as for the guiding-centre equilibrium dynamics $\{\cdot, \cdot\}_{0}$ with strong toroidal flow $\boldsymbol{u}_{0}[19]$. We will denote this Poisson bracket simply by $\{\cdot, \cdot\}$ in the following. The collisionless gyrokinetic equation is given by

$$
\frac{d F}{d t}=\frac{\partial F}{\partial t}+\{F, H\}=0
$$

We write the total distribution (in gyrocentre coordinates) in the form $F=F_{0}+\delta f$ as a sum of equilibrium and perturbation. Similarly, the Hamiltonian is written in terms of its equilibrium part $H_{0}$ and the perturbation $\bar{H}_{1}$. Then, to order $O\left(\delta^{0}\right)$ in $\delta$ :

$$
\frac{d_{0} F_{0}}{d t} \equiv \frac{\partial F_{0}}{\partial t}+\left\{F_{0}, H_{0}\right\}=0,
$$


expressing the fact that the equilibrium distribution function $F_{0}$ is a function of the constants of the unperturbed motion, $E, P_{\phi}, \mu$. To first order $O(\delta)$, we obtain

$$
\frac{d_{0} \delta f}{d t} \equiv \frac{\partial \delta f}{\partial t}+\left\{\delta f, H_{0}\right\}=-\left\{F_{0}, \bar{H}_{1}\right\}=\left\{\bar{H}_{1}, F_{0}\right\}
$$

Writing now $F_{0}=F_{0}\left(E, P_{\phi}, \mu ; \sigma\right)$, where $\sigma$ is an additional label, allowing to distinguish between co-passing and counter-passing orbits, we find

$$
\left\{\bar{H}_{1}, F_{0}\right\}=\left\{\bar{H}_{1}, H_{0}\right\} \frac{\partial F_{0}}{\partial E}+\left\{\bar{H}_{1}, P_{\phi}\right\} \frac{\partial F_{0}}{\partial P_{\phi}}+\left\{\bar{H}_{1}, \mu\right\} \frac{\partial F_{0}}{\partial \mu} .
$$

We can simplify the first term by noting that $\mathrm{d}_{0} \bar{H}_{1} / \mathrm{d} t=\partial_{t} \bar{H}_{1}+\left\{\bar{H}_{1}, H_{0}\right\}$, so that

$$
\left\{\bar{H}_{1}, H_{0}\right\}=\frac{\mathrm{d}_{0} \bar{H}_{1}}{\mathrm{~d} t}-\frac{\partial \bar{H}_{1}}{\partial t} .
$$

Furthermore, it is easy to show from a general property of Lagrangians with symmetries that

$$
\left\{\cdot, P_{\phi}\right\}=\frac{\partial}{\partial \phi}, \quad\{\cdot, \mu\} \rightleftharpoons \frac{q}{m} \frac{\partial}{\partial \zeta},
$$

because the toroidal angle $\phi$ is conjugate to $P_{\phi}$ and the gyroangle $\zeta$ is conjugate to the gyroaction $J=m \mu / q$. We thus find

$$
\frac{\mathrm{d}_{0} \delta f}{\mathrm{~d} t}=\left(\frac{\mathrm{d}_{0} \bar{H}_{1}}{\mathrm{~d} t}-\frac{\partial \bar{H}_{1}}{\partial t}\right) \frac{\partial F_{0}}{\partial E}+\frac{\partial \bar{H}_{1}}{\partial \phi} \frac{\partial F_{0}}{\partial P_{\phi}},
$$

having used that $\bar{H}_{1}$ is gyro-angle independent to eliminate the $\partial / \partial \zeta$ term. We may equivalently write this in terms of the non-adiabatic part

as

$$
\delta h \equiv \delta f-\bar{H}_{1} \frac{\partial F_{0}}{\partial E},
$$

$$
\frac{\mathrm{d}_{0} \delta h}{\mathrm{~d} t}=\frac{\partial \bar{H}_{1}}{\partial \phi} \frac{\partial F_{0}}{\partial P_{\phi}}-\frac{\partial \bar{H}_{1}}{\partial t} \frac{\partial F_{0}}{\partial E} .
$$

A similar equation for the non-adiabatic part has previously been found by Porcelli et al. [13] in the absence of strong flows.

\subsubsection{Pull-back to guiding-centre coordinates}

The gyrokinetic distribution, as written in gyrocentre coordinates, has now been shown to be of the form $F_{\mathrm{gy}}=F_{0}+\delta f$, where $F_{0}=F_{0}\left(P_{\phi}, E, \mu ; \sigma\right)$ is a function of the constants of motion. The corresponding distribution in guiding-centre coordinates, is given by $F_{\text {gc }}=F_{\text {gy }}+\overline{\boldsymbol{G}}_{1} \cdot \mathrm{d} F_{\text {gy }}+O\left(\delta^{2}\right)$, or more explicitly

$$
F_{\mathrm{gc}}=F_{0}+\delta f+\bar{G}_{1}^{P_{\phi}} \frac{\partial F_{0}}{\partial P_{\phi}}+\bar{G}_{1}^{E} \frac{\partial F_{0}}{\partial E}+\bar{G}_{1}^{\mu} \frac{\partial F_{0}}{\partial \mu},
$$


with $\bar{G}_{1}^{a}$ given by eq. $(29)$. The component $\bar{G}_{1}^{P_{\phi}}$ is found from

$$
\bar{G}_{1}^{P_{\phi}}=\overline{\boldsymbol{G}}_{1} \cdot d P_{\phi}=\boldsymbol{e}_{\phi} \cdot\left(\overline{\boldsymbol{G}}_{1} \cdot \mathrm{d} \Gamma_{0}\right)=\boldsymbol{e}_{\phi} \cdot\left(\Gamma_{1}+\mathrm{d} \bar{S}_{1}\right)=\Gamma_{1 \phi}+\frac{\partial \bar{S}_{1}}{\partial \phi}
$$

Similarly, we find

$$
\bar{G}_{1}^{\mu}=\overline{\boldsymbol{G}}_{1} \cdot d \mu=\boldsymbol{e}_{\zeta} \cdot\left(\overline{\boldsymbol{G}}_{1} \cdot \mathrm{d} \Gamma_{0}\right)=\boldsymbol{e}_{\zeta} \cdot\left(\Gamma_{1}+\mathrm{d} \bar{S}_{1}\right)=\Gamma_{1 \zeta}+\frac{\partial \bar{S}_{1}}{\partial \zeta}
$$

Finally, $\bar{G}_{1}^{E}$ is determined from the relation $\bar{H}_{1}=H_{1}-\bar{G}_{1} \cdot \mathrm{d} H_{0}=H_{1}-\bar{G}_{1}^{E}$ :

$$
\bar{G}_{1}^{E}=H_{1}-\bar{H}_{1} \text {. }
$$

Using these expressions and writing $\delta f=\bar{H}_{1} \partial F_{0} / \partial E+\delta h$, we obtain the general expression (valid to arbitrary order in $k_{\perp} \rho$ ):

$$
F_{\mathrm{gc}}=F_{0}+\bar{G}_{1}^{P_{\phi}} \frac{\partial F_{0}}{\partial P_{\phi}}+H_{1} \frac{\partial F_{0}}{\partial E}+\bar{G}_{1}^{\mu} \frac{\partial F_{0}}{\partial \mu}+\delta h .
$$

The non-adiabatic perturbed distribution function $\delta h$ is given by equation 30 .

Note that Helander et al. [31] have argued that the perturbed distribution $\delta f$ should be of the form $\delta f=-\delta \boldsymbol{I} \cdot \frac{\partial F_{0}}{\partial \boldsymbol{I}}$, where $\delta \boldsymbol{I}=\left(\delta \mathcal{E}, \delta \mathcal{P}_{\phi}, \delta \mu\right)$ are the perturbed constants of motion including adiabatic and non-adiabatic contributions. In [31], it is also claimed that this is true to arbitrary order in $\epsilon_{B}$. This is in accordance with our results based on modern gyro-kinetic theory. In fact, our derivation shows that this is the case even in the presence of a strong electric field at equilibrium (which was assumed to vanish in [31]). Furthermore, from our derivation following a Lie perturbation two-step reduction (to guiding-centre coordinates, then to gyro-centre coordinates), we have an algorithm to compute higher-order corrections in $\epsilon_{B}$.

\subsubsection{Long-wavelength limit $\epsilon_{B} \sim k_{\perp} \rho \ll 1$}

In the long-wavelength limit, we have $\tilde{F}_{\text {gc }} \ll\left\langle F_{\text {gc }}\right\rangle$. By direct calculation, we find $\left\langle\bar{G}_{1}^{P_{\phi}}\right\rangle \approx q \delta A_{\phi},\left\langle H_{1}\right\rangle \approx q \delta \phi$, and

$$
\left\langle\bar{G}_{1}^{\mu}\right\rangle \approx\left\langle q \boldsymbol{\rho} \cdot \nabla \delta \boldsymbol{A} \cdot \partial_{\zeta} \boldsymbol{\rho}\right\rangle=-\mu \frac{\delta B_{\|}}{B} .
$$

Therefore, in the long-wavelength limit where we are justified to drop oscillatory contributions in gyroangle, one obtains to leading order:

$$
F_{\mathrm{gc}}=F_{0}+q \delta A_{\phi} \frac{\partial F_{0}}{\partial P_{\phi}}+q \delta \phi \frac{\partial F_{0}}{\partial E}-\mu \frac{\delta B_{\|}}{B} \frac{\partial F_{0}}{\partial \mu}+\delta h,
$$

where the non-adiabatic contribution $\delta h$ is a solution of the drift-kinetic equation

with

$$
\begin{aligned}
\frac{\mathrm{d}_{0} \delta h}{\mathrm{~d} t} & =\frac{\partial \bar{H}_{\mathrm{dk}}}{\partial \phi} \frac{\partial F_{0}}{\partial P_{\phi}}-\frac{\partial \bar{H}_{\mathrm{dk}}}{\partial t} \frac{\partial F_{0}}{\partial E} . \\
\bar{H}_{\mathrm{dk}} & \equiv q \delta \phi-q \delta \boldsymbol{A} \cdot \dot{\boldsymbol{X}}+\mu \delta B_{\|} .
\end{aligned}
$$

These equations allow for strong flows on the order of ion sound velocity, and in this respect generalize the result of Porcelli et al [13], despite the calculation being more compact. 


\subsubsection{Consistency with non-linear guiding-centre equations}

To show that the linearized kinetic equation derived in the previous section is consistent with the linear limit of the non-linear guiding-centre Lagrangian of section 4.1, we briefly consider the linearization of (3). To this end, we write $\mathcal{L}$ in the form $\mathcal{L}=\mathcal{L}_{0}+\delta \mathcal{L}$, where $\mathcal{L}_{0}$ is the equilibrium Lagrangian and $\delta \mathcal{L}$ collects all perturbed terms, and is given by

$$
\delta \mathcal{L}=[q \delta \boldsymbol{A}+m \delta \boldsymbol{W}] \cdot \mathrm{d} \boldsymbol{X}-\left[m \delta \boldsymbol{W} \cdot \boldsymbol{W}_{0}+\mu \delta B_{\|}+q \delta \phi\right] \mathrm{d} t .
$$

We have introduced the convenient notation $\delta \boldsymbol{W} \equiv w \delta \boldsymbol{b}+\delta \boldsymbol{u}$, which is the perturbed counter-part of of $\boldsymbol{W}_{0}=w \boldsymbol{b}+\boldsymbol{u}_{0}$. By a near-identity phase-space transformation to "Hamiltonian" coordinates, we can achieve that the Lagrangian written in these new "Hamiltonian" coordinates takes the form

$$
\overline{\delta \mathcal{L}}=\left\{[q \delta \boldsymbol{A}+m \delta \boldsymbol{W}] \cdot \frac{\mathrm{d}_{0} \boldsymbol{X}}{\mathrm{d} t}-\left[m \delta \boldsymbol{W} \cdot \boldsymbol{W}_{0}+\mu \delta B_{\|}+q \delta \phi\right]\right\} \mathrm{d} t .
$$

We are thus able to move the perturbation from the symplectic part to the Hamiltonian part of the Lagrangian.

Writing $\dot{\boldsymbol{X}}_{0} \equiv \mathrm{d}_{0} \boldsymbol{X} / \mathrm{d} t$, we can express the perturbed Lagrangian in Hamiltonian coordinates as

$$
\begin{aligned}
\overline{\delta \mathcal{L}} & =-\left[q \delta \phi-q \delta \boldsymbol{A} \cdot \dot{\boldsymbol{X}}_{0}+\mu \delta B_{\|}\right] \mathrm{d} t-\left[m \delta \boldsymbol{W} \cdot\left(\dot{\boldsymbol{X}}_{0}-\boldsymbol{W}_{0}\right)\right] \mathrm{d} t \\
& =-\left[q \delta \phi-q \delta \boldsymbol{A} \cdot \dot{\boldsymbol{X}}_{0}+\mu \delta B_{\|}\right] \mathrm{d} t+O\left(\epsilon_{B}^{2}\right)
\end{aligned}
$$

having taken into account that $m \delta \boldsymbol{W} \sim \epsilon_{B} q \delta \boldsymbol{A} \cdot \dot{\boldsymbol{X}}_{0}$ and $\dot{\boldsymbol{X}}_{0}=\boldsymbol{W}_{0}+O\left(\epsilon_{B}\right)$ in the last equality. Clearly, we then have $\overline{\delta \mathcal{L}}=-\bar{H}_{\mathrm{dk}} \mathrm{d} t$ in the long-wavelength limit (33), and the kinetic equation $(32)$ can be recovered also from guiding-centre theory.

\subsubsection{Quasi-neutrality}

We now show how to recover the leading-order quasi-neutrality equation first derived by Antonsen, Lee [14]. Note that (31) expresses the pull-back in terms of $F_{0}=F_{0}\left(P_{\phi}, E, \mu\right)$. Taking $F_{0}$ to be a function of $\psi^{*} \equiv-P_{\phi} / q, \mathcal{E}$, instead, we find from $\mathcal{E}=E-q \Phi\left(\psi^{*}\right)$ :

Using

$$
\begin{gathered}
\left.\frac{\partial F_{0}}{\partial E}\right|_{P_{\phi}}=\left.\frac{\partial F_{0}}{\partial \mathcal{E}}\right|_{\psi^{*}}, \\
\left.q \frac{\partial F_{0}}{\partial P_{\phi}}\right|_{E}=-\left.\frac{\partial F_{0}}{\partial \psi^{*}}\right|_{E}=-\left.\frac{\partial F_{0}}{\partial \psi^{*}}\right|_{\mathcal{E}}-\left.\left.\frac{\partial \mathcal{E}}{\partial \psi^{*}}\right|_{E} \frac{\partial F_{0}}{\partial \mathcal{E}}\right|_{\psi^{*}} . \\
\left.\frac{\partial \mathcal{E}}{\partial \psi^{*}}\right|_{E}=-q \frac{\mathrm{d} \Phi_{0}}{\mathrm{~d} \psi},
\end{gathered}
$$

and choosing the ideal MHD gauge $\delta \boldsymbol{A}=\boldsymbol{\xi} \times \boldsymbol{B}$, we have

$$
\delta A_{\phi}=[\boldsymbol{\xi} \times \boldsymbol{B}]_{\phi}=\xi^{\psi},
$$


1

2

3

4

5

6

7

8

and obtain

$$
\delta f=-\left.\xi^{\psi} \frac{\partial F_{0}}{\partial \psi^{*}}\right|_{\mathcal{E}}+\left.q\left(\delta \phi+\xi^{\psi} \frac{\mathrm{d} \Phi_{0}}{\mathrm{~d} \psi}\right) \frac{\partial F_{0}}{\partial \mathcal{E}}\right|_{\psi^{*}}+\delta h .
$$

Writing $\delta \phi=-\xi^{\psi} \frac{\mathrm{d} \Phi_{0}}{\mathrm{~d} \psi}+\delta \phi_{L}$, where $\delta \phi_{L}$ is the "Lagrangian" perturbation, we can express this equivalently in the form

$$
\delta f=-\left.\xi^{\psi} \frac{\partial F_{0}}{\partial \psi^{*}}\right|_{\mathcal{E}}+\left.q \delta \phi_{L} \frac{\partial F_{0}}{\partial \mathcal{E}}\right|_{\psi^{*}}+\delta h .
$$

The result of Antonsen and Lee [14] is now recovered by making the approximation $\psi^{*} \approx \psi$ in the pull-back relation, so that

$$
\xi^{\psi} \frac{\partial F_{0}}{\partial \psi^{*}} \approx \boldsymbol{\xi} \cdot \nabla F_{0},
$$

where $F_{0} \approx F_{0}(\psi, \mathcal{E})$. We can then express the pull-back (31) in the form

$$
\delta f \approx-\boldsymbol{\xi}_{\perp} \cdot \nabla F_{0}+q \delta \phi_{L} \frac{\partial F_{0}}{\partial \mathcal{E}}+\delta h .
$$

Integrating over velocity space and summing over species, we find the Lagrangian perturbation

$$
0=\sum_{s} q_{s} \delta n_{s}=\sum_{s} q_{s} \int \mathrm{d}^{3} \boldsymbol{v}_{\mathrm{gc}} \delta h_{s}+\delta \phi_{L} \sum_{s} q_{s}^{2} \int \mathrm{d}^{3} \boldsymbol{v}_{\mathrm{gc}} \frac{\partial F_{0 s}}{\partial \mathcal{E}},
$$

where we have assumed quasi-neutrality at equilibrium $\sum_{s} q_{s} n_{0 s}=0$. For Maxwellian distributions, $\partial F_{0} / \partial \mathcal{E}=-1 / T_{0}$, so that the leading-order quasi-neutrality equation leads to

$$
\delta \phi_{L}=\rho_{\Phi}^{-1} \sum_{s} q_{s} \int \mathrm{d}^{3} \boldsymbol{v}_{\mathrm{gc}} \delta h_{s}
$$

where $\rho_{\Phi} \equiv \sum_{s} q_{s}^{2} n_{0 s} / T_{0 s}$. As explained below, in section $4.4 . \delta \phi_{L}$ directly determines the parallel electric field (measured in a frame moving with $\boldsymbol{u}_{0}$ ). Therefore, (34) corrects ideal Ohm's law.

Clearly, 35 is only the leading-order expression for $\delta \phi_{L}$. From gyrokinetics, we know that it is necessary to retain polarization drift effects in the quasi-neutrality equation, in order to correctly reproduce the dispersion relation for drift-waves from gyrokinetic theory 32]. Let us therefore indicate how such additional FLR corrections may be obtained from the present approach. In taking the long-wavelength limit in section 4.3.4 we have dropped the gyro-angle dependent contribution $\widetilde{F}_{\mathrm{gc}}$ to the guiding-centre distribution. If we retain this contribution, then we find to the required accuracy

$$
\widetilde{F}_{\mathrm{gc}} \approx q(\boldsymbol{\rho} \cdot \nabla) \xi^{\psi} \frac{\partial F_{0}}{\partial P_{\phi}}+q(\boldsymbol{\rho} \cdot \nabla) \delta \phi \frac{\partial F_{0}}{\partial E} \approx-(\boldsymbol{\rho} \cdot \nabla) \xi^{\psi} \frac{\partial F_{0}}{\partial \psi^{*}}+q(\boldsymbol{\rho} \cdot \nabla) \delta \phi_{L} \frac{\partial F_{0}}{\partial \mathcal{E}},
$$

where $F_{\text {gc }}=\left\langle F_{\text {gc }}\right\rangle+\widetilde{F}_{\text {gc }}$. Correspondingly, instead of the quasi-neutrality relation (34), we now obtain an additional term in the general expression for the physical number density of species $s$,

$$
\begin{aligned}
n_{s}(\boldsymbol{r}) & =\int \mathrm{d}^{3} \boldsymbol{v}_{\mathrm{gc}} F_{\mathrm{gc}} \delta(\boldsymbol{X}+\boldsymbol{\rho}-\boldsymbol{r}) \\
& \approx \int \mathrm{d}^{3} \boldsymbol{v}_{\mathrm{gc}}\left\langle F_{\mathrm{gc}}\right\rangle \delta(\boldsymbol{X}+\boldsymbol{\rho}-\boldsymbol{r})+\int \mathrm{d}^{3} \boldsymbol{v}_{\mathrm{gc}} \widetilde{F}_{\mathrm{gc}}(\boldsymbol{\rho} \cdot \nabla \delta(\boldsymbol{X}-\boldsymbol{r})) .
\end{aligned}
$$


After an integration by parts in the last term on the right, we now have to retain an additional term in the quasi-neutrality relation, which arises due to the $\boldsymbol{\rho} \cdot \nabla \delta \phi_{L^{-}}$ correction in $\widetilde{F}_{\text {gc }}$ :

$$
\begin{aligned}
0= & \sum_{s} q_{s} \int \mathrm{d}^{3} \boldsymbol{v}_{\mathrm{gc}} \delta h_{s} \delta\left(\boldsymbol{X}+\boldsymbol{\rho}_{0}-\boldsymbol{r}\right)+\left(\sum_{s} q_{s}^{2} \int \mathrm{d}^{3} \boldsymbol{v}_{\mathrm{gc}} \frac{\partial F_{0 s}}{\partial \mathcal{E}}\right) \delta \phi_{L} \\
& -\nabla \cdot\left[\left(\sum_{s} q_{s}^{2} \int \mathrm{d}^{3} \boldsymbol{v}_{\mathrm{gc}} \boldsymbol{\rho}_{0} \boldsymbol{\rho}_{0} \frac{\partial F_{0 s}}{\partial \mathcal{E}}\right) \cdot \nabla \delta \phi_{L}\right] .
\end{aligned}
$$

For Maxwellian distribution functions, and neglecting the FLR-correction due to electrons, this can be re-written in the form

$$
\begin{aligned}
0= & \sum_{s} q_{s} \int \mathrm{d}^{3} \boldsymbol{v}_{\mathrm{gc}} \delta h_{s} \delta\left(\boldsymbol{X}+\boldsymbol{\rho}_{0}-\boldsymbol{r}\right)-\left(\sum_{s} \frac{q_{s}^{2} n_{0 s}}{T_{0 s}}\right) \delta \phi_{L} \\
& +\nabla \cdot\left(\frac{q_{i}^{2} n_{0 i}}{T_{0 i}} \rho_{i}^{2} \nabla_{\perp} \delta \phi_{L}\right),
\end{aligned}
$$

where $\rho_{i} \equiv \sqrt{m_{i} T_{0 i}} /\left(q_{i} B\right)$ denotes the thermal gyroradius of the ions. Equation (36) is the relevant quasi-neutrality relation including FLR-corrections.

As seen from (36), the additional corrections appear at order $\left(k_{\perp} \rho_{i}\right)^{2} \sim \epsilon_{B}{ }^{2}$ in the long-wavelength limit. As we have argued in section 4.1.2, the kinetic-MHD model only requires guiding-centre corrections up to order $\epsilon_{B}$ to consistently provide pressure closure in the ordered momentum equation including diamagnetic effects (26). In addition, as explained in the same section 4.1.2, the current density needs to be known to order $\epsilon_{B}^{2}$ to consistently close the momentum equation. We recall that $\delta \boldsymbol{j}$ is obtained from $\mu_{0} \delta \boldsymbol{j}=\nabla \times \delta \boldsymbol{B}$, and $\delta \boldsymbol{B}$ is found from Faraday's law

$$
-\frac{\partial \delta \boldsymbol{B}}{\partial t}=\nabla \times \delta \boldsymbol{E} .
$$

Crucially for the present approach, the electrostatic contribution $\nabla \delta \phi$ to $\delta \boldsymbol{E}$ cancels in the above equation. Therefore, the calculation of $\delta \boldsymbol{B}$ (and $\delta \boldsymbol{j}$ ) to the required higher order does also not require knowledge of higher-order corrections to $\delta \phi_{L}$. Thus, owing to the convenient cancellation of the electric field upon summation of the momentum equation over species (using quasi-neutrality), and thanks to the cancellation of the electrostatic contribution in Faraday's law, the consideration of higher-order corrections in (36) does not appear to be necessary within the kinetic-MHD approach followed in the present work. Consistent with the above observations, the authors of [34] have used the leading-order form of the quasi-neutrality relation (34) to study the combined effects of diamagnetic flows and parallel electric field, based on a self-consistent kinetic-MHD model with bounce-averaged kinetic closure for trapped particles. In addition to resolving MHD instabilities, the model of Ref. [34] also obtained electrostatic instabilities, and the coupling between them.

The convenient cancellations in a kinetic-MHD approach are thus to be contrasted with a purely kinetic approach, where a consistent treatment would have required us to be able to derive from reduced kinetic equations all the necessary terms in the mo- 
mentum equation ${ }^{4}$

$$
m_{s} n_{s}\left(\frac{\partial \overline{\boldsymbol{u}}_{s}}{\partial t}+\overline{\boldsymbol{u}}_{s} \cdot \nabla \overline{\boldsymbol{u}}_{s}\right)=-\nabla \cdot \boldsymbol{P}_{s}+\epsilon_{B}{ }^{-1} q_{s} n_{s}\left(\boldsymbol{E}+\overline{\boldsymbol{u}}_{s} \times \boldsymbol{B}\right) .
$$

In this case, we would then clearly have had to retain second-order corrections to $\boldsymbol{E} \approx \boldsymbol{E}^{(0)}+\epsilon_{B} \boldsymbol{E}^{(1)}+\epsilon_{B}^{2} \boldsymbol{E}^{(2)}$ (and hence higher-order corrections to $\delta \phi_{L}$ ), in order to consistently include the required diamagnetic terms (entering at order $\epsilon_{B}$ ) in equation (37). The inclusion of such effects within a purely guiding-centre kinetic approach would consequently require the derivation (and solution) of the guiding-centre equations including all $\epsilon_{B}{ }^{2}$-corrections, a formidable task!

\subsection{Linear kinetic-MHD model including centrifugal and diamagnetic effects}

A non-linear form of the kinetic-MHD model has been presented in section 4.1. In this section, we will propose a more refined, linear kinetic-MHD model for a twocomponent plasma consisting of thermal electrons (e) and thermal ions (i), and selfconsistently taking into account centrifugal, diamagnetic as well as kinetic effects due to e.g. precession resonance. Furthermore, the model also allows the effects due to a parallel electric field to be taken into account via the solution of a quasi-neutrality equation. We will assume perturbed quantities have the following time- and toroidal dependencies $\delta X \sim \exp (-i \omega t+i n \phi)$. In section 4.2, we have shown that, under the assumption of Maxwellian distributions for $s=i, e$, the kinetic equilibrium is consistent with MHD equilibria allowing for strong flows. A drift-kinetic equation to determine the linear kinetic response to fluctuating electro-magnetic fields, and including finite-orbit width effects as well as resonances has been presented in section 4.3.4. We next want to combine the kinetic derivation with suitable fluid equations in a linear kinetic-MHD model suitable to study the stability properties of strongly flowing plasmas.

Frieman and Rotenberg [18] were the first to consider the hydrodynamic stability of strongly flowing plasmas within ideal MHD. Their derivation of the linearized stability equations is based on a Lagrangian displacement $\boldsymbol{\xi}$, which measures the difference between fluid elements advected by the perturbed and equilibrium flows. In [18, it was found that - in contrast to the stability problem in ideal MHD for a static plasma which is written in terms of a Hermitian operator - the corresponding operator for stationary plasmas with strong flows is non-Hermitian, admitting a much more complex structure of the spectrum. More recently, Aiba [23] has extended the Frieman-Rotenberg approach to include diamagnetic effects in addition to strong flows. The results of 23$]$ are obtained under the assumption of a closure relation for the pressure analogous to the one employed in ideal MHD. In this section, we will combine the kinetic equation with a version of a Frieman-Rotenberg-like equation including diamagnetic effects [23], to obtain a linear MHD model with kinetic closure suitable to study the combined diamagnetic and centrifugal effects.

We now follow largely [23], but we include a Lagrangian perturbation $\delta \phi_{L} \neq 0$ as well as a kinetic expression for the pressure tensor. We use perpendicular Ohm's law

$$
\boldsymbol{E}_{\perp}+\boldsymbol{u} \times \boldsymbol{B}=0,
$$

\footnotetext{
${ }^{4}$ Here written in non-linear form for notational convenience.
} 
for the lowest-order (MHD-)velocity $\boldsymbol{u}$, and impose $\delta \boldsymbol{B}=\nabla \times\left(\boldsymbol{\xi}_{\perp} \times \boldsymbol{B}\right)$ in terms of a new variable $\boldsymbol{\xi}$, which will be identified as the displacement. Correspondingly, we choose the gauge $\delta \boldsymbol{A}=\boldsymbol{\xi}_{\perp} \times \boldsymbol{B}$ and we find $\delta \boldsymbol{E}=-\partial \boldsymbol{\xi}_{\perp} / \partial t \times \boldsymbol{B}-\nabla \delta \phi$. With this choice of gauge, we obtain the following expression for the perturbed electric field $\delta \boldsymbol{E}^{\prime}$, as measured in a frame co-moving with $\boldsymbol{u}_{0}$ :

$$
\delta \boldsymbol{E}^{\prime} \equiv \delta \boldsymbol{E}+\boldsymbol{u}_{0} \times \delta \boldsymbol{B}=-\nabla \delta \phi_{L}-\left[\frac{\partial \boldsymbol{\xi}}{\partial t}+\boldsymbol{u}_{0} \cdot \nabla \boldsymbol{\xi}-\boldsymbol{\xi} \cdot \nabla \boldsymbol{u}_{0}\right] \times \boldsymbol{B}
$$

Note in particular that $\delta \boldsymbol{E}_{\|}^{\prime}=-\nabla_{\|} \delta \phi_{L}$ is uniquely determined by $\delta \phi_{L}$. Thus, with the present choice of gauge, a non-zero $\delta \phi_{L}$ is directly related to a non-vanishing parallel electric field $\delta \boldsymbol{E}_{\|}^{\prime} \neq 0$. This is in stark contrast to a conventional gyrokinetic treatment, which is often based on variables $\left(\delta \phi, \delta A_{\|}\right)$. We note that the precise relation between the present formulation in terms of $\left(\boldsymbol{\xi}, \delta \phi_{L}\right)$ and a formulation in terms of $\left(\delta \phi, \delta A_{\|}\right)$ appears to be a challenging open problem.

Continuing with the derivation of the linearized fluid equations, we can use the relation (38) to express the perturbed (Eulerian) MHD-velocity $\delta \boldsymbol{u}$ by re-writing the perturbed form of perpendicular Ohm's law

$$
\delta \boldsymbol{E}_{\perp}+\delta \boldsymbol{u} \times \boldsymbol{B}+\left[\boldsymbol{u}_{0} \times \delta \boldsymbol{B}\right]_{\perp}=0,
$$

as follows

$$
\delta \boldsymbol{u}_{\perp}=\left[\frac{\partial \boldsymbol{\xi}_{\perp}}{\partial t}+\left(\boldsymbol{u}_{0} \cdot \nabla\right) \boldsymbol{\xi}_{\perp}-\left(\boldsymbol{\xi}_{\perp} \cdot \nabla\right) \boldsymbol{u}_{0}\right]_{\perp}+\frac{\nabla \delta \phi_{L} \times \boldsymbol{B}}{B^{2}} .
$$

This establishes a relation for $\boldsymbol{\xi}_{\perp}$, but still leaves $\boldsymbol{\xi}_{\|}$undefined ${ }^{5}$ We will define the parallel component $\boldsymbol{\xi}_{\|}$, by requiring without loss of generality that $\delta \boldsymbol{u}$ be conveniently expressed as

$$
\delta \boldsymbol{u}=\frac{\partial \boldsymbol{\xi}}{\partial t}+\left(\boldsymbol{u}_{0} \cdot \nabla\right) \boldsymbol{\xi}-(\boldsymbol{\xi} \cdot \nabla) \boldsymbol{u}_{0}+\frac{\nabla \delta \phi_{L} \times \boldsymbol{B}}{B^{2}} .
$$

The corresponding Lagrangian velocity perturbation is then seen to be given by $\delta \boldsymbol{u}_{L}=$ $\frac{\partial \boldsymbol{\xi}}{\partial t}+\left(\boldsymbol{u}_{0} \cdot \nabla\right) \boldsymbol{\xi}+\left(\nabla \delta \phi_{L} \times \boldsymbol{B}\right) / B^{2}$, generalizing the corresponding result in [23, eq. (34)] to include an electrostatic contribution.

Based on the derivation in 23], but allowing for a perturbed pressure tensor with kinetic effects, and parallel electric fields, the following extended Frieman-Rotenberg equation for $\boldsymbol{\xi}$ is derived from this form of the Lagrangian displacement:

$$
\begin{aligned}
& \begin{array}{c}
\rho_{0}\left[\frac{\partial^{2} \boldsymbol{\xi}}{\partial t^{2}}+2\left(\boldsymbol{u}_{0} \cdot \nabla\right) \frac{\partial \boldsymbol{\xi}}{\partial t}+\left(\boldsymbol{u}_{0, * i} \cdot \nabla\right) \frac{\partial \boldsymbol{\xi}_{\perp}}{\partial t}\right] \\
+\rho_{0}\left[\frac{\partial}{\partial t}+\left(\left\{\boldsymbol{u}_{0}+\boldsymbol{u}_{0, * i}\right\} \cdot \nabla\right)\right]\left[\frac{\nabla \delta \phi_{L} \times \boldsymbol{B}}{B^{2}}\right]
\end{array} \\
& +\nabla \cdot \delta \boldsymbol{P}^{\mathrm{CGL}}-\boldsymbol{j} \times \delta \boldsymbol{B}-\delta \boldsymbol{j} \times \boldsymbol{B} \\
& =\nabla \otimes\left[\rho_{0} \boldsymbol{\xi} \otimes\left(\boldsymbol{u}_{0} \cdot \nabla\right) \boldsymbol{u}_{0}-\rho_{0} \boldsymbol{u}_{0} \otimes\left(\boldsymbol{u}_{0} \cdot \nabla\right) \boldsymbol{\xi}\right] .
\end{aligned}
$$

\footnotetext{
${ }^{5}$ When centrifugal effects are neglected, the parallel displacement is not required for the kinetic-MHD problem. The potential $\delta \phi$ and the quasi-neutrality equation replaces $\xi_{\|}$and parallel momentum equation for the MHD problem. When centrifugal effects are important, parallel and perpendicular flows cannot be trivially decoupled.
} 
Here, we have defined the ion diamagnetic drift velocity as

$$
\rho_{0} \boldsymbol{u}_{0, * i} \equiv \frac{1}{\Omega_{c i}} \boldsymbol{b} \times \nabla p_{0, i},
$$

with $\Omega_{c i}=q_{i} B / m_{i}$ the ion cyclotron frequency, and we have retained fluid diamagnetic effects only in the inertia where they are most important. This is justified if the additional diamagnetic force terms [23, e.g. eq. (40)] are dominated by either the fluid force terms, or the kinetic pressure correction arising in $\nabla \cdot \delta \boldsymbol{P}^{\mathrm{CGL}}$. Based on analytic theory in the large aspect-ratio limit, we would indeed expect kinetic corrections to dominate these diamagnetic fluid corrections, as has been shown explicitly for internal $1 / 1 \mathrm{kink}$ modes [33]. More precisely, we have neglected an additional force term [23, eq. (43)]

$$
\rho_{0}\left[\left\{(\boldsymbol{\xi} \cdot \nabla) \boldsymbol{u}_{0, * i}-\left(\boldsymbol{u}_{0, * i} \cdot \nabla\right) \boldsymbol{\xi}+\delta \boldsymbol{u}_{* i}\right\} \cdot \nabla\right] \boldsymbol{u}_{0, \perp}
$$

and similarly, we have replaced $\overline{\boldsymbol{u}}_{0}=\boldsymbol{u}_{0}+\boldsymbol{u}_{0, * i}$ by $\boldsymbol{u}_{0}$ on the right-hand side of equation (39.).

To close the momentum equation $(39)$, we note that $\delta \boldsymbol{B}=\nabla \times\left(\boldsymbol{\xi}_{\perp} \times \boldsymbol{B}\right)$ and the perturbed current is given by $\delta \boldsymbol{j}=\nabla \times \delta \boldsymbol{B} / \mu_{0}$. Finally, the perturbed CGLcontributions to the pressure are given by

with

$$
\delta \boldsymbol{P}^{\mathrm{CGL}}=\delta p_{\|} \boldsymbol{b} \boldsymbol{b}+\delta p_{\perp}(\boldsymbol{I}-\boldsymbol{b} \boldsymbol{b}),
$$

Here, the perturbed distribution function

$$
\delta f_{s}=\delta f_{s, a}+\delta h_{s}
$$

for $s=i, e$ is given in terms of an adiabatic contribution $\delta f_{s, a}$ and a non-adiabatic contribution $\delta h_{s}$. For the adiabatic contribution, we have found

$$
\delta f_{s, a}=-\left.\xi^{\psi} \frac{\partial F_{0, s}}{\partial \psi^{*}}\right|_{\mathcal{E}}+\left.q \delta \phi_{L} \frac{\partial F_{0, s}}{\partial \mathcal{E}}\right|_{\psi^{*}}
$$

To express the non-adiabatic contribution, we first note that [33]

$$
\left\langle\left.\frac{\partial F_{0}}{\partial E}\right|_{P_{\phi}}=\left.\frac{\partial F_{0}}{\partial \mathcal{E}}\right|_{\psi^{*}},\left.\quad \frac{\partial F_{0}}{\partial P_{\phi}}\right|_{E}=-\left.\frac{1}{q} \frac{\partial F_{0}}{\partial \psi^{*}}\right|_{\mathcal{E}}-\left.\left.\frac{\partial F_{0}}{\partial \mathcal{E}}\right|_{\psi^{*}} \frac{1}{q} \frac{\partial E}{\partial \psi^{*}}\right|_{\mathcal{E}} .\right.
$$

The drift-kinetic equation (33) can now be expressed in the form

$$
\frac{\mathrm{d}_{0} \delta h_{s}}{\mathrm{~d} t}=i\left(\omega-n \Omega-n \omega_{*, s}\right) \frac{\partial F_{0, s}}{\partial \mathcal{E}} \bar{H}_{\mathrm{dk}}
$$

where $\Omega=\Omega\left(\psi^{*}\right)=\mathrm{d} \Phi_{0} /\left.\mathrm{d} \psi\right|_{\psi=\psi^{*}}$ is a contribution due to the background flow and the sum of the first two terms $\omega_{D} \equiv \omega-n \Omega$ represents a Doppler shifted frequency. The third contribution $\omega_{*, s} \equiv \frac{1}{q_{s}} \frac{\partial F_{0, s}}{\partial \psi^{*}} / \frac{\partial F_{0, s}}{\partial \mathcal{E}}$ is a diamagnetic rotation frequency. Imposing 
the ideal MHD gauge $\delta \boldsymbol{A}=\boldsymbol{\xi}_{\perp} \times \boldsymbol{B}$, we can equivalently write the perturbed driftkinetic Hamiltonian $(33)$ in the form

$$
\begin{aligned}
\bar{H}_{\mathrm{dk}}= & q \delta \phi_{L}+\left[m w^{2}-\mu B\right] \boldsymbol{\xi}_{\perp} \cdot \boldsymbol{\kappa}-\mu B\left(\nabla \cdot \boldsymbol{\xi}_{\perp}\right) \\
& -\boldsymbol{\xi}_{\perp} \cdot\left[m\left(\boldsymbol{u}_{0} \cdot \nabla\right) \boldsymbol{u}_{0}+2 m w(\boldsymbol{b} \cdot \nabla) \boldsymbol{u}_{0}\right],
\end{aligned}
$$

where we have used several vector identities and $\nabla \cdot \boldsymbol{u}_{0}=0$. Equations (44), (45) extend [33, eq. (12)], taking into account centrifugal and Coriolis effects.

The full system is written in terms of $\boldsymbol{\xi}, \delta h_{s}$ and $\delta \phi_{L}$. It consists of: the momentum equation (39) to determine $\boldsymbol{\xi}$; the closure relations (40)-(43); the kinetic equation (44) for $\delta h_{s}$, which is expressed in terms of the Hamiltonian (33); and the quasi-neutrality equation (34) for $\delta \phi_{L}$. Note that we require from the kinetic equation three even moments: perpendicular pressure, parallel pressure and density. The density is required only in the quasi-neutrality relation.

Thus, we have arrived at the linear counterpart to the non-linear model of section 4.1. In contrast to the non-linear model, this linear model now includes the effects due to a non-vanishing parallel electric field $\delta \boldsymbol{E}_{\|} \neq 0$.

\subsubsection{Linear model with weak flows}

In the case of weakly flowing plasmas, centrifugal and Coriolis forces can be neglected. Nevertheless retaining weak flow and $\delta \phi_{L}$ rotation, the momentum equation (39) is conveniently written in terms of $\left(\boldsymbol{\xi}_{\perp}, \delta h_{s}\right)$, because the parallel component $\boldsymbol{\xi}_{\|}$is decoupled from $\boldsymbol{\xi}_{\perp}$ in this case. Indeed, we then find

$$
\begin{aligned}
\rho_{0}\left[\left(\frac{\partial}{\partial t}+\boldsymbol{u}_{0, * i} \cdot \nabla\right) \frac{\partial \boldsymbol{\xi}_{\perp}}{\partial t}\right. & \left.+\left(\frac{\partial}{\partial t}+\boldsymbol{u}_{0, * i} \cdot \nabla\right) \frac{\nabla \delta \phi_{L} \times \boldsymbol{B}}{B^{2}}\right] \\
& =-\left[\nabla \cdot \delta \boldsymbol{P}^{\mathrm{CGL}}\right]_{\perp}+[\boldsymbol{j} \times \delta \boldsymbol{B}]_{\perp}+\delta \boldsymbol{j} \times \boldsymbol{B} .
\end{aligned}
$$

with $\delta \boldsymbol{j}=\nabla \times \delta \boldsymbol{B} / \mu_{0}$ and $\delta \boldsymbol{B}=\nabla \times\left(\boldsymbol{\xi}_{\perp} \times \boldsymbol{B}\right)$, and $\delta \boldsymbol{P}^{\mathrm{CGL}}$ is given by (40)-(43) as a function of $\boldsymbol{\xi}_{\perp}, \delta h_{s}, \delta \phi_{L}$. The latter variables are determined from the solution of the kinetic equation (without centrifugal effects)

$$
\frac{\mathrm{d}_{0} \delta h_{s}}{\mathrm{~d} t}=i\left(\omega-n \Omega-n \omega_{*, s}\right) \frac{\partial F_{0, s}}{\partial \mathcal{E}} \bar{H}_{\mathrm{dk}}
$$

where $\omega_{*, s} \equiv \frac{1}{q_{s}} \frac{\partial F_{0, s}}{\partial \psi_{*}^{*}} / \frac{\partial F_{0, s}}{\partial \mathcal{E}}$. The perturbed drift-kinetic Hamiltonian is given by

$$
\bar{H}_{\mathrm{dk}}=q \delta \phi_{L}+\left[m w^{2}-\mu B\right] \boldsymbol{\xi}_{\perp} \cdot \boldsymbol{\kappa}-\mu B\left(\nabla \cdot \boldsymbol{\xi}_{\perp}\right) .
$$

The quasi-neutrality equation reads

$$
\left(\sum_{s} \frac{q_{s}^{2} n_{0 s}}{T_{0 s}}\right) \delta \phi_{L}=\sum_{s} q_{s} \int \mathrm{d}^{3} \boldsymbol{v}_{\mathrm{gc}} \delta h_{s}
$$

These equations are identical to those of reference [33], except that in [33] the parallel electric field was neglected $\left(\delta \phi_{L}=0\right)$. In the system of equations of [33], it was found that weak flows do affect the kinetic corrections to MHD if the plasma rotation is sheared. 
The system of equations (46)-49) is expressed in terms of $\boldsymbol{\xi}_{\perp}, \delta h_{s}$ and $\delta \phi_{L}$ and can be used to study electrostatic effects on global MHD instabilities [34]. A very similar model has previously been proposed in [14, 34], but in contrast to [14] the derivation (from gyrokinetics) adopted in this work makes no restriction on the allowed bouncefrequencies and includes full finite-orbit width effects. In addition [14, 34 neglected diamagnetic corrections in the inertia, and by neglecting finite orbit width effects in solving for the non-adiabatic part $\delta h$, the kinetic inertia effects described in 33$]$ were also not included.

\subsubsection{Linear model with strong flows, without parallel electric field}

In the presence of strong equilibrium flows, the equations for $\boldsymbol{\xi}_{\perp}$ and $\boldsymbol{\xi}_{\|}$are no longer decoupled and the parallel dynamics can have an important effect on the stability analysis. Neglecting diamagnetic and parallel electric field $\left(\delta \phi_{L}=0\right)$, but retaining kinetic wave-particle interaction, we arrive at the following model: The kinetic-MHD momentum equation (39) now becomes a Frieman-Rotenberg-like equation with kinetic closure

$$
\begin{aligned}
& \rho_{0}\left[\frac{\partial^{2} \boldsymbol{\xi}}{\partial t^{2}}+2\left(\boldsymbol{u}_{0} \cdot \nabla\right) \frac{\partial \boldsymbol{\xi}}{\partial t}\right]= \\
& \quad-\nabla \cdot \delta \boldsymbol{P}^{\mathrm{CGL}}+\boldsymbol{j} \times \delta \boldsymbol{B}+\delta \boldsymbol{j} \times \boldsymbol{B} \\
& \quad+\nabla \otimes\left[\rho_{0} \boldsymbol{\xi} \otimes\left(\boldsymbol{u}_{0} \cdot \nabla\right) \boldsymbol{u}_{\theta}-\rho_{0} \boldsymbol{u}_{0} \otimes\left(\boldsymbol{u}_{0} \cdot \nabla\right) \boldsymbol{\xi}\right] .
\end{aligned}
$$

with $\delta \boldsymbol{j}=\nabla \times \delta \boldsymbol{B} / \mu_{0}$ and $\delta \boldsymbol{B}=\nabla \times(\boldsymbol{\xi} \times \boldsymbol{B})$, and $\delta \boldsymbol{P}^{\mathrm{CGL}}$ is given by (40)-(43) as a function of $\boldsymbol{\xi}, \delta h_{s}$ where we set $\delta \phi_{L}=0$. The $\delta h_{s}$ are determined from the solution of the kinetic equation (44). In this limit, the model is expressed in terms of $\boldsymbol{\xi}$ and $\delta h_{s}$, and it allows to study the combined kinetic and centrifugal effects on MHD modes.

\section{Summary}

A kinetic-MHD model has been derived from a consistent set of guiding-centre equations. The proposed kinetic-MHD model allows for strong flows and includes centrifugal as well as FLR effects related to diamagnetic flows. Closure of the momentum equation is obtained from a solution of the guiding-centre equations, thus accounting for finite orbit-width effects and particle-wave interactions such as precession resonance.

For the first time, the full expression for the gyroviscous contribution to the pressure tensor has been obtained from kinetic theory, without resorting to any simplifying assumptions on the background geometry. Our detailed calculations demonstrate that the formulation of guiding-centre theory proposed in this work can be used to study the influence of kinetic effects on global MHD modes on time-scales $\omega_{D} \sim \omega_{*, i}$ when the diamagnetic drift frequency is of the order of the Doppler shifted rotation frequency $\omega_{D}=\omega-n \Omega$.

We have explicitly shown that for a two-component plasma $(s=i, e)$, and under the assumption of Maxwellian equilibrium distribution functions, the usual ideal MHD equilibrium equations are obtained from guiding-centre theory.

Linearized equations describing the evolution of long-wavelength $\left(k_{\perp} \rho_{i} \ll 1\right)$ global (collisionless) kinetic-MHD modes have been obtained from a kinetic extension of the 
Frieman-Rotenberg equations, allowing for centrifugal effects, diamagnetic fluid drift, as well as a kinetic closure. The model naturally includes a drift-kinetic form of the quasi-neutrality equation, and allows the effects of a parallel electric field on global MHD modes to be studied self-consistently.

In closing, we mention that while the results of this work have focused on the collisionless kinetic effects due to thermal species in a two-component plasma, an additional population of supra-thermal ions (or electrons) can be added trivially in the pressure coupling scheme, provided that either the suprathermal distributions are isotropic, or that their contributions to pressure gradients are weak.

The numerical implementation of the proposed linear model is currently underway, and the implications of the centrifugal, diamagnetic and electrostatic effects discussed in the present work, will be the subject of future publications. The proposed model can also serve as a starting point for analytical work, and is suitable for the derivation of a dispersion relation taking into account the centrifugal effects on the kinetic response of thermal ions.

\section{Acknowledgment}

This work has been carried out within the framework of the EUROfusion Consortium and has received funding from the Euratom research and training programme 2014 - 2018 and 2019 - 2020 under grant agreement No 633053. The views and opinions expressed herein do not necessarily reflect those of the European Commission. This work was supported in part by the Swiss National, Science Foundation.

\section{A FLR correction (I) - evaluation of eq. (13)}

We evaluate the contribution of

$$
\int \mathrm{d}^{3} \boldsymbol{v} m w\left[\boldsymbol{b} \boldsymbol{V}_{\mathrm{gc}}+\boldsymbol{V}_{\mathrm{gc}} \boldsymbol{b}\right] F .
$$

where we re-write eq. (8) (see also [20, eq. (49)]) in the form

$$
\boldsymbol{V}_{\mathrm{gc}}=\boldsymbol{b} \times\left[\frac{\mu}{q B} \nabla B+\frac{w^{2}}{\Omega} \boldsymbol{b} \cdot \nabla \boldsymbol{b}\right]+\frac{w}{\Omega} \boldsymbol{b} \times\left[\frac{d \boldsymbol{b}}{d t}+\boldsymbol{b} \cdot \nabla \boldsymbol{u}\right]+\frac{1}{\Omega} \boldsymbol{b} \times \frac{d \boldsymbol{u}}{d t} .
$$

and $\frac{d}{d t} \equiv \frac{\partial}{\partial t}+\boldsymbol{u} \cdot \nabla$. The relevant contribution of this correction to $\boldsymbol{P}$, is therefore

$$
\Omega P_{\boldsymbol{b} \perp}=\boldsymbol{b} \times\left[P_{\|}\left(\frac{d \boldsymbol{b}}{d t}+\boldsymbol{b} \cdot \nabla \boldsymbol{u}\right)+q_{\|}^{\perp} \nabla B+2 q_{\|}^{\|} \boldsymbol{\kappa}\right] .
$$

\section{B FLR correction (II) - evaluation of eq. (14)}

We evaluate

$$
\int \mathrm{d}^{3} \boldsymbol{v} m\left[\dot{\boldsymbol{\rho}}^{(0)} \dot{\boldsymbol{\rho}}^{(1)}+\dot{\boldsymbol{\rho}}^{(1)} \dot{\boldsymbol{\rho}}^{(0)}\right] F
$$


This term requires the evaluation of the first order correction to $\dot{\boldsymbol{\rho}}$. We have

$$
\begin{aligned}
\dot{\boldsymbol{\rho}} & =\left(\partial_{t}+\dot{\boldsymbol{X}} \cdot \nabla+\dot{w} \partial_{w}+\dot{\zeta} \partial_{\zeta}\right) \boldsymbol{\rho} \\
& =\dot{\zeta}^{(0)} \partial_{\zeta} \boldsymbol{\rho}_{0}+\epsilon_{B} \dot{\zeta}^{(1)} \partial_{\zeta} \boldsymbol{\rho}_{0}+\epsilon_{B} \partial_{t} \boldsymbol{\rho}_{0}+\epsilon_{B} \dot{\boldsymbol{X}}^{(0)} \cdot \nabla \boldsymbol{\rho}_{0}+\epsilon_{B} \dot{\zeta}^{(0)} \partial_{\zeta} \boldsymbol{\rho}_{1}
\end{aligned}
$$

We note that

$$
\boldsymbol{\rho}_{0}=\rho_{0} \widehat{\boldsymbol{\rho}}=\sqrt{\frac{2 \mu B(\boldsymbol{X}, t)}{m}} \widehat{\boldsymbol{\rho}}(\boldsymbol{X}, \zeta, t)
$$

so that

$$
\begin{aligned}
\nabla \boldsymbol{\rho}_{0} & =\frac{1}{2} \nabla \log B \otimes \boldsymbol{\rho}_{0}+\rho_{0} \nabla \widehat{\boldsymbol{\rho}}, \\
\partial_{t} \boldsymbol{\rho}_{0} & =\frac{1}{2} \partial_{t} \log B \boldsymbol{\rho}_{0}+\rho_{0} \partial_{t} \widehat{\boldsymbol{\rho}} .
\end{aligned}
$$

Furthermore,

$$
\dot{\zeta}^{(0)}=\Omega, \quad \dot{\zeta}^{(1)}=\boldsymbol{R} \cdot \boldsymbol{W}+S+\frac{1}{2} \boldsymbol{b} \cdot \nabla \times \boldsymbol{W},
$$

with $\boldsymbol{R} \equiv(\nabla \widehat{\perp}) \cdot \widehat{\boldsymbol{\rho}}=\left(\nabla \boldsymbol{e}_{1}\right) \cdot \boldsymbol{e}_{2}, S=\widehat{\boldsymbol{\rho}} \cdot \partial_{t} \widehat{\perp}=\boldsymbol{e}_{2} \cdot \partial_{t} \boldsymbol{e}_{1}$, and $\boldsymbol{W} \equiv \boldsymbol{u}+w \boldsymbol{b}$. We can now write

$$
\begin{aligned}
\frac{1}{\rho_{0}} \boldsymbol{\rho}_{1}= & {[\boldsymbol{R} \cdot \boldsymbol{W}+S] \widehat{\perp}+\left[\frac{1}{2} \boldsymbol{b} \cdot \nabla \times \boldsymbol{W}\right] \widehat{\perp}+\Omega \partial_{\zeta} \boldsymbol{\rho}_{1} / \rho_{0} } \\
& +\frac{1}{2}\left[\left(\partial_{t}+\boldsymbol{W} \cdot \nabla\right) \log B\right] \widehat{\boldsymbol{\rho}}+\left(\partial_{t}+\boldsymbol{W} \cdot \nabla\right) \hat{\boldsymbol{\rho}} .
\end{aligned}
$$

We can further simplify this expression, by noting that

$$
\begin{aligned}
{\left[\partial_{t}+\boldsymbol{W} \cdot \nabla\right] \widehat{\boldsymbol{\rho}} } & =-\left(\left[\partial_{t}+\boldsymbol{W} \cdot \nabla\right] \widehat{\perp} \cdot \widehat{\boldsymbol{\rho}}\right) \widehat{\perp}-\left(\left[\partial_{t}+\boldsymbol{W} \cdot \nabla\right] \boldsymbol{b} \cdot \widehat{\boldsymbol{\rho}}\right) \boldsymbol{b} \\
& =-[S+\boldsymbol{W} \cdot \boldsymbol{R}] \widehat{\perp}-\left(\left[\frac{d \boldsymbol{b}}{d t}+w \boldsymbol{\kappa}\right] \cdot \widehat{\boldsymbol{\rho}}\right) \boldsymbol{b} .
\end{aligned}
$$

Thus, we find that the terms involving $\boldsymbol{R}, S$ cancel, leaving an expression that is written in terms of derivatives of $\boldsymbol{B}$ and $\boldsymbol{u}$ : 
We then find, using $\widehat{\boldsymbol{\rho}}=\boldsymbol{b} \times \widehat{\perp}$ and hence $\boldsymbol{A} \cdot \widehat{\boldsymbol{\rho}}=-\boldsymbol{b} \times \boldsymbol{A} \cdot \hat{\perp}$, that

$$
\begin{aligned}
m\left\langle\dot{\boldsymbol{\rho}}^{(0)} \dot{\boldsymbol{\rho}}^{(1)}\right\rangle= & \frac{2 \mu B}{\Omega}\left\langle\widehat{\perp} \dot{\boldsymbol{\rho}}^{(1)} / \rho_{0}\right\rangle \\
= & \frac{\mu B}{\Omega}[\boldsymbol{b} \cdot \nabla \times \boldsymbol{W}]\langle\hat{\perp} \widehat{\perp}\rangle+(2 \mu B)\left\langle\widehat{\boldsymbol{\rho}} \boldsymbol{\rho}_{1} / \rho_{0}\right\rangle \\
& +\frac{\mu B}{\Omega}\left[\left(\partial_{t}+\boldsymbol{W} \cdot \nabla\right) \log B\right]\langle\hat{\perp} \widehat{\boldsymbol{\rho}}\rangle \\
& +\frac{2 \mu B}{\Omega} \boldsymbol{b} \times\left[\frac{d \boldsymbol{b}}{d t}+w \boldsymbol{\kappa}\right] \cdot\langle\widehat{\perp} \widehat{\perp}\rangle \boldsymbol{b} \\
= & \frac{\mu B}{\Omega} \frac{1}{2}[\boldsymbol{b} \cdot \nabla \times \boldsymbol{W}](\boldsymbol{I}-\boldsymbol{b} \boldsymbol{b})+(2 \mu B)\left\langle\widehat{\boldsymbol{\rho}} \boldsymbol{\rho}_{1} / \rho_{0}\right\rangle \\
& +(\mathrm{AS})+\frac{\mu B}{\Omega}\left(\boldsymbol{b} \times\left[\frac{d \boldsymbol{b}}{d t}+w \boldsymbol{\kappa}\right]\right) \boldsymbol{b},
\end{aligned}
$$

where (AS) is an anti-symmetric term, which will cancel in the final expression for the pressure, which is symmetric. To complete this calculation, we still need to evaluate $\left\langle\widehat{\boldsymbol{\rho}} \boldsymbol{\rho}_{1}\right\rangle / \rho_{0}$. Since this expression is a gyro-average, we only require the contributions to $\boldsymbol{\rho}_{1}$, which can be written as odd polynomials in $\widehat{\boldsymbol{\rho}}, \widehat{\mathcal{I}}$.

In general, we have the following expression [21]

$$
\boldsymbol{\rho}_{1}=-G_{2}^{\boldsymbol{X}}+\frac{1}{2}\left(G_{1}^{\mu} \partial_{\mu}+G_{1}^{\zeta} \partial_{\zeta}\right) G_{1}^{\boldsymbol{X}}+\frac{1}{2} G_{1}^{\boldsymbol{X}} \cdot \nabla G_{1}^{\boldsymbol{X}},
$$

where $G_{1}^{\boldsymbol{X}}=-\boldsymbol{\rho}_{0}$. The perpendicular component of $G_{2}^{\boldsymbol{X}}$ can be written [20, eq. (A17)]

$$
G_{2, \perp}^{\boldsymbol{X}}=\frac{1}{2}\left(g_{\mu} \partial_{\mu}+g_{\zeta} \partial_{\zeta}\right) \boldsymbol{\rho}_{0}+\frac{1}{\Omega}(\boldsymbol{b} \cdot \nabla \times \boldsymbol{W}) \boldsymbol{\rho}_{0},
$$

where

$$
\begin{aligned}
& G_{1}^{\mu}=g_{\mu}+\mu \boldsymbol{\rho}_{0} \cdot \nabla \log B \\
& G_{1}^{\zeta}=g_{\zeta}-\boldsymbol{\rho}_{0} \cdot \boldsymbol{R}
\end{aligned}
$$

We find that

$$
\begin{aligned}
\boldsymbol{\rho}_{1}= & -G_{2, \|}^{\boldsymbol{X}} \boldsymbol{b}-\left(g_{\mu} \partial_{\mu}+g_{\zeta} \partial_{\zeta}\right) \boldsymbol{\rho}_{0}-\frac{1}{\Omega}(\boldsymbol{b} \cdot \nabla \times \boldsymbol{W}) \boldsymbol{\rho}_{0} \\
& -\frac{1}{2} \mu\left(\boldsymbol{\rho}_{0} \cdot \nabla \log B\right) \partial_{\mu} \boldsymbol{\rho}_{0}+\frac{1}{2}\left(\boldsymbol{\rho}_{0} \cdot \boldsymbol{R}\right) \partial_{\zeta} \boldsymbol{\rho}_{0}+\frac{1}{2} \boldsymbol{\rho}_{0} \cdot \nabla \boldsymbol{\rho}_{0} \\
= & -G_{2, \|}^{\boldsymbol{X}} \boldsymbol{b}-\left(g_{\mu} \partial_{\mu}+g_{\zeta} \partial_{\zeta}\right) \boldsymbol{\rho}_{0}-\frac{1}{\Omega}(\boldsymbol{b} \cdot \nabla \times \boldsymbol{W}) \boldsymbol{\rho}_{0} \\
& -\frac{1}{4}\left(\boldsymbol{\rho}_{0} \cdot \nabla \log B\right) \boldsymbol{\rho}_{0}+\frac{1}{2}\left(\boldsymbol{\rho}_{0} \cdot \boldsymbol{R}\right) \partial_{\zeta} \boldsymbol{\rho}_{0}+\frac{1}{2} \boldsymbol{\rho}_{0} \cdot \nabla \boldsymbol{\rho}_{0}
\end{aligned}
$$

By similar manipulations as in equation (53), we observe that the last term can be written

$$
\frac{\rho_{0}^{2}}{2}[(\widehat{\boldsymbol{\rho}} \cdot \boldsymbol{R}) \hat{\perp}+\widehat{\boldsymbol{\rho}} \cdot \nabla \widehat{\boldsymbol{\rho}}]=-\frac{\rho_{0}^{2}}{2} \widehat{\boldsymbol{\rho}} \cdot \nabla \boldsymbol{b} \cdot \widehat{\boldsymbol{\rho}}=-\frac{1}{2} \boldsymbol{\rho}_{0} \cdot \nabla \boldsymbol{b} \cdot \boldsymbol{\rho}_{0}
$$


and thus

$$
\begin{aligned}
\boldsymbol{\rho}_{1}= & -\left(g_{\mu} \partial_{\mu}+g_{\zeta} \partial_{\zeta}\right) \boldsymbol{\rho}_{0}-\frac{1}{\Omega}(\boldsymbol{b} \cdot \nabla \times \boldsymbol{W}) \boldsymbol{\rho}_{0} \\
& -\left(G_{2, \|}^{\boldsymbol{X}}+\frac{1}{2} \boldsymbol{\rho}_{0} \cdot \nabla \boldsymbol{b} \cdot \boldsymbol{\rho}_{0}\right) \boldsymbol{b} .
\end{aligned}
$$

This expression is still completely general, and recovers equation (49) 21] in the limit $\boldsymbol{u} \rightarrow 0$.

Citing the results of [20] equations (A33), (A35), we find

$$
\begin{gathered}
G_{2, \|}^{\boldsymbol{X}}=-\frac{1}{\Omega} \boldsymbol{\rho}_{0} \cdot \boldsymbol{b} \times\left[\boldsymbol{b} \cdot \nabla \boldsymbol{W}+\boldsymbol{W} \cdot \nabla \boldsymbol{b}+\partial_{t} \boldsymbol{b}\right] \\
\quad-\frac{\mu}{4 q \Omega}[\hat{\boldsymbol{\rho}} \widehat{\boldsymbol{\rho}}-\widehat{\perp} \widehat{\perp}]: \nabla \boldsymbol{b} \\
g_{\mu}=-\frac{\mu}{\Omega} \boldsymbol{b} \cdot \nabla \times \boldsymbol{W}+\frac{m}{B} \boldsymbol{\rho}_{0} \cdot[(\boldsymbol{W} \cdot \nabla) \boldsymbol{W}] \\
\quad+\frac{\mu}{2 \Omega}(\widehat{\boldsymbol{\rho}} \hat{\perp}+\widehat{\perp} \hat{\boldsymbol{\rho}}): \nabla \boldsymbol{W}+\frac{m}{B} \boldsymbol{\rho}_{0} \cdot \partial_{t} \boldsymbol{W}, \\
g_{\zeta}=-\frac{q}{m} \partial_{\mu} s_{3} \\
=-\frac{1}{4 \Omega}(\widehat{\boldsymbol{\rho}} \widehat{\boldsymbol{\rho}}-\hat{\perp} \hat{\perp}): \nabla \boldsymbol{W} \\
+\boldsymbol{\rho}_{0} \cdot \frac{\boldsymbol{b} \times \nabla B}{B}-\frac{q}{2 \mu} \frac{1}{\Omega} \boldsymbol{\rho}_{0} \cdot \boldsymbol{b} \times\left[\left(\partial_{t}+\boldsymbol{W} \cdot \nabla\right) \boldsymbol{W}\right] .
\end{gathered}
$$

We only require the contribution to $G_{2, \mid}^{\boldsymbol{X}}$ that is linear in $\widehat{\boldsymbol{\rho}}, \hat{\perp}$ and the contributions to $g_{\mu}, g_{\zeta}$ which are constant or quadratic in $\hat{\boldsymbol{\rho}}, \hat{\boldsymbol{\Lambda}}$. We can therefore take

$$
\begin{aligned}
G_{2, \|}^{\boldsymbol{X}} & \rightarrow-\frac{1}{\Omega} \boldsymbol{\rho}_{0} \cdot \boldsymbol{b} \times\left[\boldsymbol{b} \cdot \nabla \boldsymbol{W}+\boldsymbol{W} \cdot \nabla \boldsymbol{b}+\partial_{t} \boldsymbol{b}\right] \\
g_{\mu} /(2 \mu) & \rightarrow-\frac{1}{2 \Omega} \boldsymbol{b} \cdot \nabla \times \boldsymbol{W}+\frac{1}{4 \Omega}(\widehat{\boldsymbol{\rho}} \widehat{\perp}+\widehat{\perp} \widehat{\boldsymbol{\rho}}): \nabla \boldsymbol{W}, \\
g_{\zeta} & \rightarrow-\frac{1}{4 \Omega}(\widehat{\boldsymbol{\rho}} \widehat{\boldsymbol{\rho}}-\hat{\perp} \hat{\perp}): \nabla \boldsymbol{W},
\end{aligned}
$$

\footnotetext{
${ }^{6}$ We here correct two mistakes in 20, eq. (A35)]: The first term in that reference has an erroneous additional $\frac{1}{2}$, the second term does not gyro-average to zero in [20].
} 
when carrying out the gyroaverage $\left\langle\widehat{\boldsymbol{\rho}} \boldsymbol{\rho}_{1} / \rho_{0}\right\rangle$.

$$
\begin{aligned}
& \left\langle\widehat{\boldsymbol{\rho}} \boldsymbol{\rho}_{1} / \rho_{0}\right\rangle=-\left\langle g_{\mu} /(2 \mu) \hat{\boldsymbol{\rho}} \widehat{\boldsymbol{\rho}}\right\rangle-\left\langle g_{\zeta} \widehat{\boldsymbol{\rho}} \hat{\perp}\right\rangle \\
& -\frac{1}{\Omega}(\boldsymbol{b} \cdot \nabla \times \boldsymbol{W})\langle\widehat{\boldsymbol{\rho}} \widehat{\boldsymbol{\rho}}\rangle-\left\langle G_{2, \|}^{\boldsymbol{X}} \widehat{\boldsymbol{\rho}}\right\rangle \boldsymbol{b} \\
& =\frac{1}{2 \Omega}(\boldsymbol{b} \cdot \nabla \times \boldsymbol{W})\langle\hat{\boldsymbol{\rho}} \widehat{\boldsymbol{\rho}}\rangle+\frac{1}{\Omega}\left\langle\widehat{\boldsymbol{\rho}} \widehat{\boldsymbol{\rho}}\left[-\frac{(\widehat{\boldsymbol{\rho}} \hat{\perp}+\hat{\perp} \widehat{\boldsymbol{\rho}})}{4}\right]: \nabla \boldsymbol{W}\right\rangle \\
& +\frac{1}{\Omega}\left\langle\hat{\boldsymbol{\rho}} \hat{\perp}\left[\frac{(\widehat{\boldsymbol{\rho}} \hat{\boldsymbol{\rho}}-\hat{\perp} \hat{\perp})}{4}\right]: \nabla \boldsymbol{W}\right\rangle-\frac{1}{\Omega}(\boldsymbol{b} \cdot \nabla \times \boldsymbol{W})\langle\hat{\boldsymbol{\rho}} \hat{\boldsymbol{\rho}}\rangle \\
& -\frac{1}{\Omega}\langle\widehat{\boldsymbol{\rho}} \widehat{\boldsymbol{\rho}}\rangle \cdot\left(\boldsymbol{b} \times\left[\boldsymbol{b} \cdot \nabla \boldsymbol{W}+\boldsymbol{W} \cdot \nabla \boldsymbol{b}+\frac{1}{2} \partial_{t} \boldsymbol{b}\right]\right) \boldsymbol{b} \\
& =-\frac{1}{4 \Omega}(\boldsymbol{b} \cdot \nabla \times \boldsymbol{W})(\boldsymbol{I}-\boldsymbol{b} \boldsymbol{b}) \\
& +\frac{1}{\Omega}\left\langle\hat{\boldsymbol{\rho}} \widehat{\boldsymbol{\rho}}\left[-\frac{(\widehat{\boldsymbol{\rho}} \hat{\perp}+\hat{\perp} \hat{\boldsymbol{\rho}})}{4}\right]: \nabla \boldsymbol{W}\right\rangle \\
& +\frac{1}{\Omega}\left\langle\widehat{\boldsymbol{\rho}} \hat{\perp}\left[\frac{(\widehat{\boldsymbol{\rho}} \hat{\boldsymbol{\rho}}-\hat{\perp} \hat{\perp})}{4}\right]: \nabla \boldsymbol{W}\right\rangle \\
& -\frac{1}{2 \Omega}\left(\boldsymbol{b} \times\left[2 w \boldsymbol{\kappa}+\boldsymbol{b} \cdot \nabla \boldsymbol{u}+\boldsymbol{u} \cdot \nabla \boldsymbol{b}+\frac{1}{2} \partial_{t} \boldsymbol{b}\right]\right) \boldsymbol{b} .
\end{aligned}
$$

After carrying out the remaining gyro-averages, we find

$$
\begin{aligned}
& (2 \mu B)\left\langle\widehat{\boldsymbol{\rho}} \boldsymbol{\rho}_{1} / \rho_{0}\right\rangle=-\frac{\mu B}{2 \Omega}(\boldsymbol{b} \cdot \nabla \times \boldsymbol{W})(\boldsymbol{I}-\boldsymbol{b} \boldsymbol{b}) \\
& \frac{\mu B}{4 \Omega}\left[\left(\nabla_{1} W_{2}+\nabla_{2} W_{1}\right)\left(\boldsymbol{e}_{1} \boldsymbol{e}_{1}-\boldsymbol{e}_{2} \boldsymbol{e}_{2}\right)\right. \\
& \left.+\left(\nabla_{2} W_{2}-\nabla_{1} W_{1}\right)\left(\boldsymbol{e}_{1} \boldsymbol{e}_{1}+\boldsymbol{e}_{2} \boldsymbol{e}_{1}\right)\right] \\
& -\frac{\mu B}{\Omega}\left(\boldsymbol{b} \times\left[2 w \boldsymbol{\kappa}+\boldsymbol{b} \cdot \nabla \boldsymbol{u}+\boldsymbol{u} \cdot \nabla \boldsymbol{b}+\partial_{t} \boldsymbol{b}\right]\right) \boldsymbol{b} \text {. }
\end{aligned}
$$


We therefore finally have

$$
\begin{aligned}
m\left[\left\langle\dot{\boldsymbol{\rho}}^{(0)} \dot{\boldsymbol{\rho}}^{(1)}\right\rangle+\left\langle\dot{\boldsymbol{\rho}}^{(0)} \dot{\boldsymbol{\rho}}^{(1)}\right\rangle\right]= & \frac{\mu B}{\Omega}[\boldsymbol{b} \cdot \nabla \times \boldsymbol{W}](\boldsymbol{I}-\boldsymbol{b} \boldsymbol{b}) \\
& +(2 \mu B)\left[\left\langle\widehat{\boldsymbol{\rho}} \boldsymbol{\rho}^{(1)} / \rho_{0}\right\rangle+\left\langle\boldsymbol{\rho}^{(1)} \hat{\boldsymbol{\rho}} / \rho_{0}\right\rangle\right] \\
& +\frac{\mu B}{\Omega}\left[\left(\boldsymbol{b} \times\left[\frac{d \boldsymbol{b}}{d t}+w \boldsymbol{\kappa}\right]\right) \boldsymbol{b}+(T)\right] \\
= & \frac{\mu B}{2 \Omega}\left[\left(\nabla_{1} W_{2}+\nabla_{2} W_{1}\right)\left(\boldsymbol{e}_{1} \boldsymbol{e}_{1}-\boldsymbol{e}_{2} \boldsymbol{e}_{2}\right)\right. \\
& \left.\left.+\left(\nabla_{2} W_{2}-\nabla_{1} W_{1}\right)\left(\boldsymbol{e}_{1} \boldsymbol{e}_{1}+\boldsymbol{e}_{2} \boldsymbol{e}_{1}\right)\right]\right) \\
& +\frac{\mu B}{\Omega}\left[\left(\boldsymbol{b} \times\left[\frac{d \boldsymbol{b}}{d t}+w \boldsymbol{\kappa}\right]\right) \boldsymbol{b}+(T)\right] \\
& -\frac{\mu B}{\Omega}\left[\left(\boldsymbol{b} \times\left[2 w \boldsymbol{\kappa}+\boldsymbol{b} \cdot \nabla \boldsymbol{u}+\frac{d \boldsymbol{b}}{d t}\right]\right) \boldsymbol{b}+(T)\right] \\
= & \frac{\mu B}{2 \Omega}\left[\left(\nabla_{1} W_{2}+\nabla_{2} W_{1}\right)\left(\boldsymbol{e}_{1} \boldsymbol{e}_{1}-\boldsymbol{e}_{2} \boldsymbol{e}_{2}\right)\right. \\
& \left.+\left(\nabla_{2} W_{2}-\nabla_{1} W_{1}\right)\left(\boldsymbol{e}_{1} \boldsymbol{e}_{1}+\boldsymbol{e}_{2} \boldsymbol{e}_{1}\right)\right] \\
& +\frac{\mu B}{\Omega}[(\boldsymbol{b} \times[-w \boldsymbol{\kappa}-\boldsymbol{b} \cdot \nabla \boldsymbol{u}]) \boldsymbol{b}+(T)],
\end{aligned}
$$

where $(T)$ indicates the transpose of the term to its left.

\section{FLR correction (III) - evaluation of eq. (15)}

We evaluate

$$
(\mathrm{III})=-\epsilon_{B} \mathrm{~d}^{3} \boldsymbol{v} m\left[\dot{\boldsymbol{\rho}}^{(0)}\left(\boldsymbol{\rho}_{0} \cdot \nabla \boldsymbol{u}\right)+\left(\boldsymbol{\rho}_{0} \cdot \nabla \boldsymbol{u}\right) \dot{\boldsymbol{\rho}}^{(0)}\right] F
$$

We find

$$
\begin{aligned}
(\mathrm{III})=-\epsilon_{B} \int \mathrm{d}^{3} \boldsymbol{v} m\left[\dot{\boldsymbol{\rho}}\left(\boldsymbol{\rho}_{0} \cdot \nabla \boldsymbol{u}\right)+(\boldsymbol{\rho} \cdot \nabla \boldsymbol{u}) \dot{\boldsymbol{\rho}}_{0}\right] F \\
=-\epsilon_{B} \int \mathrm{d}^{3} \boldsymbol{v} \frac{2 \mu B}{\Omega}[\widehat{\perp}(\widehat{\boldsymbol{\rho}} \cdot \nabla \boldsymbol{u})+(\widehat{\boldsymbol{\rho}} \cdot \nabla \boldsymbol{u}) \widehat{\perp}] F \\
=-\epsilon_{B} \frac{p_{\perp}}{\Omega}\left[\boldsymbol{e}_{1}\left(\boldsymbol{e}_{2} \cdot \nabla \boldsymbol{u}\right)-\boldsymbol{e}_{2}\left(\boldsymbol{e}_{1} \cdot \nabla \boldsymbol{u}\right)\right. \\
\left.+\left(\boldsymbol{e}_{2} \cdot \nabla \boldsymbol{u}\right) \boldsymbol{e}_{1}-\left(\boldsymbol{e}_{1} \cdot \nabla \boldsymbol{u}\right) \boldsymbol{e}_{2}\right]
\end{aligned}
$$

This can be further simplified as

$$
\begin{aligned}
(\mathrm{III})= & \frac{P_{\perp}}{\Omega}\left[\left(\nabla_{2} u_{2}-\nabla_{1} u_{1}\right)\left(\boldsymbol{e}_{1} \boldsymbol{e}_{2}+\boldsymbol{e}_{2} \boldsymbol{e}_{1}\right)\right. \\
& \left.+\left(\nabla_{2} u_{1}+\nabla_{1} u_{2}\right)\left(\boldsymbol{e}_{1} \boldsymbol{e}_{1}-\boldsymbol{e}_{2} \boldsymbol{e}_{2}\right)\right] \\
& +\frac{P_{\perp}}{\Omega}[\boldsymbol{b} \times(\nabla \boldsymbol{u} \cdot \boldsymbol{b})+(T)] \\
& -\frac{P_{\perp}}{\Omega}(\boldsymbol{b} \cdot \nabla \times \boldsymbol{u})(\boldsymbol{I}-\boldsymbol{b} \boldsymbol{b}) .
\end{aligned}
$$




\section{FLR correction (IV) - evaluation of eq. (16)}

We evaluate

$$
\begin{aligned}
-\nabla \cdot \int \mathrm{d}^{3} \boldsymbol{v} m w \boldsymbol{\rho}_{0}\left[\boldsymbol{b} \dot{\boldsymbol{\rho}}^{(0)}+\dot{\boldsymbol{\rho}}^{(0)} \boldsymbol{b}\right] F & =-\nabla \cdot \int \mathrm{d}^{3} \boldsymbol{v} \frac{w \mu B}{\Omega} F[2\langle\hat{\boldsymbol{\rho}} \boldsymbol{b} \widehat{\perp}\rangle+2\langle\hat{\boldsymbol{\rho}} \widehat{\perp} \boldsymbol{b}\rangle] \\
& =-\nabla \cdot\left(\frac{q_{\|}^{\perp}}{\Omega}[2\langle\hat{\boldsymbol{\rho}} \boldsymbol{b} \widehat{\perp}\rangle+2\langle\widehat{\boldsymbol{\rho}} \widehat{\perp} \boldsymbol{b}\rangle]\right)
\end{aligned}
$$

and

$$
2\langle\widehat{\boldsymbol{\rho}} \boldsymbol{b} \widehat{\perp}\rangle+2\langle\widehat{\boldsymbol{\rho}} \hat{\perp} \boldsymbol{b}\rangle=\boldsymbol{e}_{1}\left(\boldsymbol{e}_{2} \boldsymbol{b}+\boldsymbol{b} \boldsymbol{e}_{2}\right)-\boldsymbol{e}_{2}\left(\boldsymbol{e}_{1} \boldsymbol{b}+\boldsymbol{b} \boldsymbol{e}_{1}\right)
$$

We have

$$
\begin{aligned}
-\nabla \cdot\left(\frac{q_{\|}^{\perp}}{\Omega}[2\langle\hat{\boldsymbol{\rho}} \boldsymbol{b} \hat{\perp}\rangle+2\langle\hat{\boldsymbol{\rho}} \hat{\perp} \boldsymbol{b}\rangle]\right)= & -\left(\frac{1}{\Omega} \nabla q_{\|}^{\perp}-\frac{q_{\|}^{\perp}}{\Omega} \nabla \log (B)\right) \cdot\left[\boldsymbol{e}_{1}\left(\boldsymbol{e}_{2} \boldsymbol{b}+\boldsymbol{b} \boldsymbol{e}_{2}\right)-\boldsymbol{e}_{2}\left(\boldsymbol{e}_{1} \boldsymbol{b}+\boldsymbol{b} \boldsymbol{e}_{1}\right)\right] \\
& -\frac{q_{\|}^{\perp}}{\Omega} \nabla \cdot\left[\boldsymbol{e}_{1}\left(\boldsymbol{e}_{2} \boldsymbol{b}+\boldsymbol{b} \boldsymbol{e}_{2}\right)-\boldsymbol{e}_{2}\left(\boldsymbol{e}_{1} \boldsymbol{b}+\boldsymbol{b} \boldsymbol{e}_{1}\right)\right] \\
= & \frac{1}{\Omega}\left(\boldsymbol{b} \times\left[\nabla q_{\|}^{\perp}-q_{\|}^{\perp} \nabla \log (B)\right]\right) \boldsymbol{b} \\
& -\frac{q_{\|}^{\perp}}{\Omega} \nabla \cdot\left[\boldsymbol{e}_{1}\left(\boldsymbol{e}_{2} \boldsymbol{b}+\boldsymbol{b} \boldsymbol{e}_{2}\right)-\boldsymbol{e}_{2}\left(\boldsymbol{e}_{1} \boldsymbol{b}+\boldsymbol{b} \boldsymbol{e}_{1}\right)\right] .
\end{aligned}
$$

Here

hence

$$
\begin{aligned}
\nabla \cdot\left[\boldsymbol{e}_{1}\left(\boldsymbol{e}_{2} \boldsymbol{b}+\boldsymbol{b} \boldsymbol{e}_{2}\right)-\boldsymbol{e}_{2}\left(\boldsymbol{e}_{1} \boldsymbol{b}+\boldsymbol{b} \boldsymbol{e}_{1}\right)\right]= & \boldsymbol{b}(\boldsymbol{b} \times \boldsymbol{\kappa})+(\boldsymbol{b} \times \boldsymbol{\kappa}) \boldsymbol{b} \\
& -2(\boldsymbol{b} \cdot \nabla \times \boldsymbol{b}) \boldsymbol{b} \boldsymbol{b}+(\boldsymbol{b} \cdot \nabla \times \boldsymbol{b})(\boldsymbol{I}-\boldsymbol{b} \boldsymbol{b}) \\
& +\left(\nabla_{1} b_{1}-\nabla_{2} b_{2}\right)\left(\boldsymbol{e}_{1} \boldsymbol{e}_{2}+\boldsymbol{e}_{2} \boldsymbol{e}_{1}\right) \\
& +\left(\nabla_{1} b_{2}+\nabla_{2} b_{1}\right)\left(\boldsymbol{e}_{2} \boldsymbol{e}_{2}-\boldsymbol{e}_{1} \boldsymbol{e}_{1}\right),
\end{aligned}
$$

$$
\begin{aligned}
-\nabla \cdot \int \mathrm{d}^{3} \boldsymbol{v} m w \boldsymbol{\rho}_{0}\left\langle\boldsymbol{b} \dot{\boldsymbol{\rho}}^{(0)}+\dot{\boldsymbol{\rho}}^{(0)} \boldsymbol{b}\right] F= & \frac{1}{\Omega} \boldsymbol{b} \times\left[\nabla q_{\|}^{\perp}-q_{\|}^{\perp} \nabla \log (B)-q_{\|}^{\perp} \boldsymbol{\kappa}\right] \boldsymbol{b} \\
& -\frac{q_{\|}^{\perp}}{\Omega}(\boldsymbol{b} \cdot \nabla \times \boldsymbol{b})(\boldsymbol{I}-\boldsymbol{b} \boldsymbol{b}) \\
& -\frac{2 q_{\|}^{\perp}}{\Omega}(\boldsymbol{b} \cdot \nabla \times \boldsymbol{b}) \boldsymbol{b} \boldsymbol{b} \\
& -\frac{q_{\|}^{\perp}}{\Omega}\left[\left(\nabla_{1} b_{1}-\nabla_{2} b_{2}\right)\left(\boldsymbol{e}_{1} \boldsymbol{e}_{2}+\boldsymbol{e}_{2} \boldsymbol{e}_{1}\right)\right. \\
& \left.+\left(\nabla_{1} b_{2}+\nabla_{2} b_{1}\right)\left(\boldsymbol{e}_{2} \boldsymbol{e}_{2}-\boldsymbol{e}_{1} \boldsymbol{e}_{1}\right)\right] .
\end{aligned}
$$

Interestingly, the contribution $\nabla \log (B)$ here exactly cancels a similar $\nabla B$ contribution from eq. (13) which is obtained as a result of the magnetic $\nabla B$ drift, so that the final expression is written in terms of $\boldsymbol{\kappa}$ and not $\nabla B$. 


\section{References}

[1] G.F. Chew, M.L. Goldberger, and F.E. Low. The Boltzmann Equation and the One-Fluid Hydromagnetic Equations in the Absence of Particle Collisions. Proc. R. Soc. London (London), A236:112, 1956.

[2] K. V. Roberts and J. B. Taylor. Magnetohydrodynamic equations for finite larmor radius. Phys. Rev. Lett., 8:197-198, Mar 1962.

[3] Z. Chang and J. D. Callen. Generalized gyroviscous force and its effect on the momentum balance equation. Physics of Fluids B: Plasma Physics, 4(7):17661771, 1992.

[4] A. I. Smolyakov. Gyroviscous forces in a collisionless plasma with temperature gradients. Can. J. Phys., 76:321-331, 1998.

[5] A. N. Kaufman. Plasma Viscosity in a Magnetic Field. Phys. Fluids, 3(4):610, 1960.

[6] A. B. Mikhailovskii and V. S. Tsypin. Transport equations and gradient instabilities in a high pressure collisional plasma. Plasma Physics, 13(9):785-798, sep 1971.

[7] R. D. Hazeltine and J. D. Meiss. Shear-Alfvén dynamics of toroidally confined plasmas. Physics Reports, 121(1-2):1-164, 1985.

[8] A. Macmahon. Finite Gyro-Radius Corrections to the Hydromagnetic Equations for a Vlasov Plasma. Physics of Fluids, 8(10):1840, 1965.

[9] J. J. Ramos. Fluid formalism for collisionless magnetized plasmas. Physics of Plasmas, 12(5):1-14, 2005.

[10] J. J. Ramos. General expression of the gyroviscous force. Physics of Plasmas, 12(11):1-7, 2005.

[11] A. J. Brizard. Nonlinear gyrofluid description of turbulent magnetized plasmas. Physics of Fluids B, 4(5):1213-1228, 1992.

[12] E. V. Belova. Nonlinear gyroviscous force in a collisionless plasma. Physics of Plasmas, 8(9):3936-3944, 2001.

[13] F. Porcelli, R. Stankiewicz, W. Kerner, and H. L. Berk. Solution of the driftkinetic equation for global plasma modes and finite particle orbit widths. Physics of Plasmas, 1(3):470-480, 1994.

[14] T. M. Antonsen. Electrostatic modification of variational principles for anisotropic plasmas. Physics of Fluids, 25(1):132, 1982.

[15] B. D. Scott. Tokamak edge turbulence: background theory and computation. Plasma Physics and Controlled Fusion, 49(7):S25-S41, jun 2007.

[16] H. Lütjens and J.-F. Luciani. XTOR-2F: A fully implicit NewtonKrylov solver applied to nonlinear 3D extended MHD in tokamaks. Journal of Computational Physics, 229(21):8130 - 8143, 2010.

[17] H. Lütjens and J.-F. Luciani. The XTOR code for nonlinear 3D simulations of MHD instabilities in tokamak plasmas. Journal of Computational Physics, 227(14):6944 - 6966, 2008. 
[18] E. Frieman and M. Rotenberg. On hydromagnetic stability of stationary equilibria. Rev. Mod. Phys., 32:898-902, Oct 1960.

[19] A. J. Brizard. Nonlinear gyrokinetic Vlasov equation for toroidally rotating axisymmetric tokamaks. Physics of Plasmas, 2(May 2014):459, 1995.

[20] J. Madsen. Second order guiding-center vlasov-maxwell equations. Physics of Plasmas, 17(8):082107, 2010.

[21] A. J. Brizard. Guiding-center polarization and magnetization effects in gyrokinetic theory. arXiv e-prints, page arXiv:1008.3888, Aug 2010.

[22] S. I. Braginskii. Transport processes in a plasma. Reviews of Plasma Physics, 1, 1965.

[23] N Aiba. Impact of ion diamagnetic drift on ideal ballooning mode stability in rotating tokamak plasmas. Plasma Physics and Controlled Fusion, 58(4):045020, 2016.

[24] M. N. Rosenbluth and N. Rostoker. Theoretical structure of plasma equations. The Physics of Fluids, 2(1):23-30, 1959.

[25] J. P. Freidberg. Ideal MHD. Cambridge University Press, 2014.

[26] S. Jolliet, A. Bottino, P. Angelino, R. Hatzky, T.M. Tran, B.F. Mcmillan, O. Sauter, K. Appert, Y. Idomura, and L. Villard. A global collisionless pic code in magnetic coordinates. Computer Physics Communications, 177(5):409 - 425, 2007.

[27] T. Görler, X. Lapillonne, S. Brunner, T. Dannert, F. Jenko, F. Merz, and D. Told. The global version of the gyrokinetic turbulence code gene. Journal of Computational Physics, 230(18):7053 - 7071, 2011.

[28] P. J. Catto, I. B. Bernstein, and M. Teśsarotto. Ion transport in toroidally rotating tokamak plasmas. The Physics of Fluids, 30(9):2784-2795, 1987.

[29] J P Graves and C Wahlberg. Generalised zonal modes in stationary axisymmetric plasmas. Plasma Physics and Controlled Fusion, 59(5):054011, 2017.

[30] A. J. Brizard and T. S. Hahm. Foundations of nonlinear gyrokinetic theory. Rev. Mod. Phys., 79:421-468, Apr 2007.

[31] P. Helander, C. G. Gimblett, R. J. Hastie, and K. G. McClements. The influence of fast ions on the magnetohydrodynamic stability of negative shear profiles. Physics of Plasmas, 4(6):2181-2187, 1997.

[32] D. Dubin, J. A. Krommes, C. Oberman, and W. Lee. Nonlinear gyrokinetic equations. The Physics of fluids, 26(12):3524-3535, 1983.

[33] J P Graves, R J Hastie, and K I Hopcraft. The effects of sheared toroidal plasma rotation on the internal kink mode in the banana regime. Plasma Physics and Controlled Fusion, 42(10):1049, 2000.

[34] T. M. Antonsen and A. Bondeson. Effects of trapped thermal particles on the $\mathrm{n}=1$ internal kink mode in tokamaks. Phys. Rev. Lett., 71:2046-2049, 1993. 